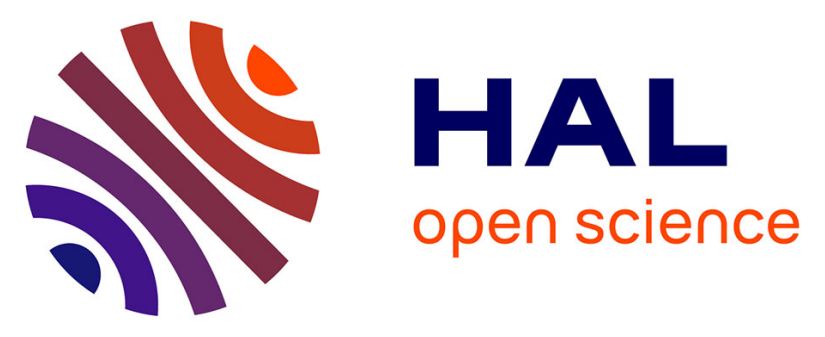

\title{
Statistical Abundance and Stability of carbon nanostructures by Combined Condensation-Annealing Molecular Dynamics simulations
}

Amal Allouch, Jonathan Mougenot, Swaminathan Prasanna, Armelle Michau, Mahamadou J Seydou, François Maurel, Pascal Brault, Khaled Hassouni

\section{To cite this version:}

Amal Allouch, Jonathan Mougenot, Swaminathan Prasanna, Armelle Michau, Mahamadou J Seydou, et al.. Statistical Abundance and Stability of carbon nanostructures by Combined CondensationAnnealing Molecular Dynamics simulations. Computational and Theoretical Chemistry, 2021, 1201, pp.113252. 10.1016/j.comptc.2021.113252 . hal-03223432

\section{HAL Id: hal-03223432 \\ https://hal.science/hal-03223432}

Submitted on 11 May 2021

HAL is a multi-disciplinary open access archive for the deposit and dissemination of scientific research documents, whether they are published or not. The documents may come from teaching and research institutions in France or abroad, or from public or private research centers.
L'archive ouverte pluridisciplinaire HAL, est destinée au dépôt et à la diffusion de documents scientifiques de niveau recherche, publiés ou non, émanant des établissements d'enseignement et de recherche français ou étrangers, des laboratoires publics ou privés. 


\title{
Statistical Abundance and Stability of carbon nanostructures by Combined Condensation-Annealing Molecular Dynamics simulations
}

\author{
$\underline{\text { A. Allouch }}^{1}$, J. Mougenot ${ }^{1}$, A. Michau ${ }^{1}$, S. Prasanna ${ }^{1}$, K . Hassouni ${ }^{1}$, \\ M. Seydou ${ }^{2}$, F. Maurel ${ }^{2}$ and P. Brault ${ }^{3}$ \\ ${ }^{1}$ Laboratoire des Sciences des Procédés et des Matériaux (LSPM), CNRS UPR 3407, \\ Université Sorbonne Paris Nord, Villetaneuse, France \\ ${ }^{2}$ Laboratoire Interfaces, Traitements, Organisation et Dynamique des Systèmes (ITODYS), CNRS UMR 7086 \\ Université Paris Diderot, France \\ ${ }^{3}$ Groupe de Recherches sur l'Energétique des Milieux Ionisés (GREMI), CNRS UMR 7344, \\ Université d'Orléans, France
}

\begin{abstract}
Molecular dynamic (MD) simulations of the Combined Condensation and Annealing (CCA) of free carbon atoms was used to investigate the statistical abundance and stability of neutral carbon nanostructures for a cluster size between 2 and 54 atoms. During these CCA MD-simulations carbon atoms are submitted to a condensation/heating phase, a CTR phase at a prescribed annealing temperature and a cooling phase. Numerical experiments showed that the determination of the statistical abundances requires at least $100 \mathrm{~ns}$ condensation/heating phase, $100 \mathrm{~ns}$ CTR phase at the annealing temperature and $100 \mathrm{~ns}$ cooling phase. The clusters obtained by CCA MD-simulations are dominated by linear structures for $n=2-5$, mono-ring for $n=6$ 15 , multi-ring structures for $\mathrm{n}=16-18,2 \mathrm{D}$ graphene-like structure for sizes in the range $\mathrm{n}=19-29$ and cage-like structure above $n=30$. Open cage structures are obtained at temperature around $2000 \mathrm{~K}$, while closed cage structures dominate at $3000 \mathrm{~K}$. For even larger temperature, i.e., $4000 \mathrm{~K}$, the dominant structures are 2D and 3D multi-ring structures, 2D graphene-like structure remaining quite significant.
\end{abstract}

Keywords: carbon clusters, Molecular Dynamics, SREBO potential, carbon condensation, carbon annealing, LAMMPS, Dusty plasma.

\section{Introduction}

Carbon clusters and nanoparticles attracted a lot of attention from both physicists and chemists over the last four decades (AJAYAN and IIJIMA, 1992; Ebbesen et al., 1992; Prassides and Kroto, 1992; Willett and Fisher, 
1992, 1992). These nanostructures show unique functional properties that can be used in a variety of advanced technological and biomedical applications (Harrison and Atala, 2007; Tang et al., 2010; Wang et al., 2015). This motivated the development and investigation of several synthesis processes among which laser vaporization and arc discharges have been probably the most used and investigated (Samsonov and Goree, 1999). Carbon nanoparticles can be also obtained in low temperature non equilibrium glow discharges such as the graphite cathode DC sputtering discharges (A Michau et al., 2016) or the capacitively coupled radiofrequency discharges (Samsonov and Goree, 1999). In these discharges, the cathode sputtering leads to the emission of a significant amount of carbon, the major part of which is in the form of carbon atoms (Bernholc and Phillips, 1986). These atoms that are usually emitted at fairly high kinetic energy, i.e., around $1 \mathrm{eV}$ (Dominique and Arnas, 2014) experience an enhanced quenching due to the collisions with the background gas phase atoms, usually argon or helium. A carbon supersaturation is subsequently achieved and the condensation of the carbon vapor takes place (Dominique and Arnas, 2014). This condensation phase consists of molecular growth processes that end up with the nucleation of solid carbon nanoparticles (A Michau et al., 2016; Armelle Michau et al., 2016).The size distribution and structure of the obtained particles strongly depend on the nucleation kinetics that is linked to the molecular growth processes and transport phenomena that govern carbon clusters (Armelle Michau et al., 2016). In particular, the nucleation kinetic strongly depends on the molecular growth processes involving the intermediate size carbon clusters, i.e., $n=10-n=50$. In fact, during the molecular growth, carbon clusters are submitted to highly non-equilibrium conditions which may result in the formation of several isomers (Alexandrov and Schweigert, n.d.). As a matter of fact, the coexistence of a significant high energy ion flux with a cold neutral background in the considered discharges may lead to very large temperature fluctuations of clusters and nanoparticles (Armelle Michau et al., 2016; Prasanna et al., 2019). The later experiences collisions with high energy ions, which leads to a strong heating followed by a rapid quenching due to collisions with the low temperature background neutrals. The authors of reference (Prasanna et al., 2019) showed that this may lead to more than $2000 \mathrm{~K}$ temperature fluctuation for $1 \mathrm{~nm}$ carbon nanoparticles. The effect is probably more pronounced for smaller clusters and temperatures as high as 3000 $\mathrm{K}$ can be reached. Such temperature can also be obtained by adsorption and coalescence of small cluster as showed in (Brault et al., 2019). These temperature fluctuations may be regarded as a dynamic annealing process during which the clusters may transition between several configurations. In such conditions, the molecular growth process for a given size depends on the statistical abundance of the different isomers. As matter of fact, molecular growth proceeds by coagulation of large clusters with atomic carbon or $\mathrm{C}_{2}$ dimer (Bernholc and Phillips, 1986; Armelle Michau et al., 2016). The coagulation probability of a given size cluster with $\mathrm{C}_{\text {or }} \mathrm{C}_{2}$ may significantly differ depending on the nature of the considered isomer for this cluster due to geometric consideration. Therefore, the knowledge of the abundance of the different isomers for each cluster size under this non equilibrium conditions is an important parameter for the understanding of nanoparticle formation and 
of the correlation between the structural and the functional characteristics of the nanoparticles. This is also necessary for the prediction of the structural characteristics of carbon nanoparticles (Arnas et al., 2010).

The investigation of carbon cluster structures with sizes ranging between few and few tens of atoms received a large attention in the literature. Basically, one may distinguish two types of studies as far as cluster formation is concerned. The first one focuses on the structural analysis and stability of both neutral and charged carbon clusters and involves ab initio, DFT and classical molecular dynamic approaches (An et al., 2005; Bleil et al., 1994; Jones, 1999; Liang and Iii, 1990). For instance, an ab-initio study of $C_{n}(n=2-10)$ performed by K. Raghavachari and J.S. Binkley (Raghavachari and Binkley, 1987) showed that the odd-numbered clusters have a linear structure while the even-numbered clusters adopt an irregular cyclic structure. A.C. Ngandjong et al. (Ngandjong et al., 2017) also used DFT to analyze the energetic of many anionic carbon clusters $C_{n}^{-}(n \leq 10)$ and showed that the most stable geometries remain linear irrespective of the cluster size. Medium cluster size, i.e., $\mathrm{C}_{20}$, and $C_{n}(n \leq 32)$, have been also investigated using a DFT approach by An et al. and Jones (An et al., 2005; Jones, 1999); respectively. Several isomers including chains, rings, fullerene-like (cage), and graphitic plate or bowl structures were found. In particular, it was shown that even with advanced method, different stable forms may be obtained depending on the adopted level of theory and on the basis set used in DFT simulations. Some groups have focused on the investigation by DFT methods of cage isomers for large carbon clusters with up to 50 atoms ( Grossman et al., 1998; Małolepsza et al., 2007; Sokolova et al., 2000; Tang et al., 2011). Most of the published works made use of DFT methods for thorough structural and topological investigations of the cage isomers for some clusters of interest such as $\mathrm{C}_{20}, \mathrm{C}_{28}, \mathrm{C}_{36}, \mathrm{C}_{60}$ and $\mathrm{C}_{62}$. Of particular interest is the work of Tang et al. (Tang et al., 2011) who used spiral algorithm for cluster cage isomers generation and DFT methods for cage geometry optimization, in order to investigate the different cage isomers of carbon clusters with sizes ranging between 30 and 50 carbons. They identified a large number of isomers, including both classical and non-classical fullerene. They showed that the number of isomers, especially the non-classical ones, increases exponentially with the cluster size. The energy spectrum of these isomers shows that although a classical fullerene; i.e. a cage containing only hexagon and pentagon, is always the more stable isomers, some of the so called non-classical isomers, i.e., those containing at least one heptagon, are more stable than some classical fullerene (Tang et al., 2011).

The structural analysis of large clusters was also performed using the classical MD approach that requires lower computational cost as compared to ab initio and DFT methods. Most of the authors used one of the several versions of Brenner potential along with an optimization algorithm to reach the structure and evaluate the energy of the more stable isomers. Hobday and Smith used a genetic algorithm in order to investigate the structure and stability of isomers for clusters with size ranging between 3 and 60 carbon atoms (Hobday and Smith, 1997). Cai et al. combined Simulated Annealing (SA) method with a genetic algorithm to obtain the 
lowest energies for carbon clusters $C_{n}$ from $n=2$ to $n=7$ (Cai et al., 2004). Lai et al. combined Monte Carlo (MC) simulations with a modified version of the so called Basin Hopping (MBH) algorithm to identify the more stable structure (Lai et al., 2017). Ballone and Milani used the Simulated Annealing (SA) approach in order to explore the potential hypersurface and determine the more stable cluster structures for cluster sizes ranging between 50 and 72 (Ballone and Milani, 1990). The same technique of simulated annealing was used by Maruyama and Yamaguchi to obtain a perfect $\mathrm{D}_{6 \mathrm{~h}}$ structure from imperfect fullerene for the $\mathrm{C}_{60}$ cluster (Maruyama and Yamaguchi, 1998). Ning and coworkers (Gao et al., 2007a; Wang et al., 2008; Xiu-Fang et al., 2008) developed a global optimization method based on time-going-backward quasi-dynamics that makes it possible to cross high energy barrier of the potential surface and reach a large number of local minima, thus enhancing the probability to reach the global minimum (Wang et al., 2008). They used this technique to determine the energy spectrum of different structures of $\mathrm{C}_{30}, \mathrm{C}_{31}$ (Xiu-Fang et al., 2008) and $\mathrm{C}_{36}$ (Gao et al., 2007a) clusters. They also derived and validated a free energy criterion for the stability of the isomers of these clusters (Gao et al., 2007a). To our best knowledge, except in the case of the work performed by Hobday and Smith (Hobday and Smith, 1997) and Gao et al. (Gao et al., 2007a), the main objective of these MD based studies was the identification of the most stable structures for a given geometry, e.g., chains, cages or graphene.

The second group of MD based studies is much more kinetic in nature. The authors focused on the investigation of carbon condensation at a prescribed temperature with a special attention to the mechanism involved in either fullerene formation from carbon vapor (Yamaguchi and Maruyama, 1998), tricyclic structure (Schwiegert, 1995) and graphite sheet (Lebedeva et al., 2008; Pietrucci and Andreoni, 2014), or carbon rich structure nucleation in interstellar media (Patra et al., 2014). In all these studies, molecular growth of carbon clusters were investigated for the relevant conditions, shedding the light on several peculiarities of the growth or transformation mechanisms. In particular, Ning and coworkers reported that during carbon structure condensation in helium buffer gas, intermediate size clusters undergo dynamic fluctuations between several structures and both planar and cage isomers may be obtained for $\mathrm{C}_{30}$ and $\mathrm{C}_{31}$ clusters (Xiu-Fang et al., 2008). This finding open up the key-question with respect to the abundance of the different form of intermediate size carbon clusters.

This is the question we address in this paper where we are interested in the statistical abundance of the different structures for intermediate carbon clusters sizes (i.e., number of carbon ranging between 10 and 54 atoms) under non equilibrium conditions characterized by temperature ramps involving a strong heating phase and a thermal quenching phase.

The paper is organized as follows. In the next section we briefly give the main feature of our MD approach and discuss the methodology in setting up the combined condensation/annealing simulation approach to 
determine the isomers abundance. The results obtained are discussed in section 3 where we present our simulation methodology, analyze the abundance of the different structures for a wide range of cluster size. Although our approach was not intended to identify the most stable structure for a given size, we investigated the more stable structures obtained with CCA MD-simulations and compare them to those obtained with several other methods dedicated to stable structure identification. The implication of this work as far as molecular growth and nanoparticle formation in glow discharge is discussed in the last section.

\section{Modeling and simulation method}

The method is based on Combined Condensation and Annealing (CCA) cycles. A given number of low temperature free carbon atoms are first randomly positioned inside a simulation box. These atoms, that undergo a rapid condensation, are submitted to a heating up to a prescribed temperature, a CTR phase at this temperature and a cooling phase. During this combined condensation and annealing, the fate of the atoms is followed by molecular dynamic simulation. It is worthy to mention here that we are not interested in the investigation of the clustering kinetic, but rather in determining the abundance of the different structural groups that may be obtained at the end of the CCA cycle. The details of the adopted interaction potentials and simulation procedure are described in the following subsections.

\subsection{Classical Molecular Dynamics simulation}

The classical MD method is based on the integration of momentum equations that are derived from Newton's law assuming that all the interaction between atoms is described by an empirical classical potential. The Second-generation Reactive Empirical Bond Order REBO (SREBO) potential (Brenner et al., 2002) that was specifically parameterized for carbon and hydrogen-carbon systems is employed to model the interatomic interactions between carbon atoms. A comparative analysis of the different interaction potentials used for carbon system was performed by Lai et al. (Lai et al., 2017) who showed that, compared to other potentials, the SREBO potential yields more accurate energy values for the different structures of several carbon clusters. This potential, that is implemented in LAMMPS software (Plimpton, 1995) (version of 22-08-2018), is a reformulation of Brenner-Tersoff potentials (Brenner, 1990; Tersoff, 1989, 1988, 1986), that are in turn based on Abell's formalism (Abell, 1985) in which the binding energy is produced by the nearest-neighbor interactions that depend on the local coordination and bond angles. SREBO allows for covalent bond breaking and formation with appropriate changes in atomic hybridization during the simulation. The analytical expression of the SREBO potential energy includes a repulsive interatomic term, an attractive interatomic term, a cutoff function, and a bond-order term denoted $f_{c}, V_{R}, V_{A}$ and $B_{i j}$ in reference (Brenner et al., 2002), respectively. The cutoff function limits the range of the covalent interactions. The bond order term includes 
the many body effects and takes into account the conjugation bonds and the torsion effects, i.e., the terms denoted $b_{i j}{ }^{\mathrm{RC}}$ and $b_{\mathrm{ij}}{ }^{\mathrm{DH}}$ in reference (Brenner et al., 2002).

\subsection{Combined condensation annealing MD-simulation}

In this work MD simulations are used to investigate the fate of a randomly distributed free carbon atoms submitted to a combined condensation/annealing transformation. The condensation phase takes place under the action of the attractive forces between the initially free carbon atoms and ends up with the formation of a carbon cluster that undergo several rearrangements during a simultaneous annealing process. The MD simulation method used in this work is a combination between the one used to describe the condensation of free carbon atoms in reference (Gao et al., 2007a; Xiu-Fang et al., 2008; Yamaguchi and Maruyama, 1998) where the authors investigated the energy spectrum of $\mathrm{C}_{30}, \mathrm{C}_{31}$ and $\mathrm{C}_{36}$ isomers and the $\mathrm{C}_{70}$ fullerene formation process, on one hand and the one used in reference (Ballone and Milani, 1990; Maruyama and Yamaguchi, 1998) where the authors investigated the annealing of imperfect carbon clusters and their transformation to a perfect $\mathrm{D}_{6 \mathrm{~h}}$ Buckminster fullerene, on the other hand. MD-simulations of CCA are used in the present work in order to estimate the statistical abundance of the different isomers for a given cluster $C_{n}(10 \leq n \leq 54)$ without any assumption with respect to the cluster geometry.

Depending on the size of the system a variety of isomers can be obtained at the end of the simulation. For small size systems, isomers can appear only in three shapes: linear, monocyclic or 2D polycyclic structures. As the size of the system increase the number of isomers exponentially increases implying a huge number for very large systems. Some studies have studied a large spectrum of carbon isomers by detailing the different isomers belonging to the same structural group. Note however that such a detailed information is not needed when one is interested to physical effects or parameters where the small structural differences between isomers belonging to the same class does not play any role. This is likely the case when one is interested in coagulation kinetics. As a matter of fact, coagulation processes with two clusters isomers that show very close structure would likely show very similar kinetics. On the opposite coagulation processes of $\mathrm{C}$ or $\mathrm{C} 2$ with two clusters with the same size but belonging to a different classis, e.g., graphene-like and cage-like, would probably show a fairly different kinetics.

For this purpose, in our study we only differentiate six structural groups: graphene-like, 2D-polycyclic, 3Dpolycyclic, bowl-like, open cage-like and closed cage-like (fullerenes). Typical structures belonging to these groups are shown in Figure 1. Graphene-like 2D clusters are essentially made of coplanar hexagonal cycles with edge defects consisting of 1-3 pentagon and/or heptagon cycles usually located at the edge of the cluster (cf. Figure 1a). 2D polycyclic groups include all the clusters made of coplanar cycles that are different form graphene-like 2D clusters (cf. Figure 1b). 3D polycyclic clusters are made of non-planar cycles (cf. Figure 1c). 
Bowl group includes spherical caps shaped clusters (cf. Figure 1d). Closed-cage group include cage clusters that contain essentially hexagon and pentagon, with possibly few defects, and are usually termed classical or non-classical fullerenes depending on the number of pentagon and hexagon rings they involve. The classical fullerenes are defined as a closed carbon cages $\mathbf{C}_{\mathbf{n}}$ containing 12 pentagons and $\left(\frac{\mathbf{n}}{\mathbf{2}}-\mathbf{1 0}\right)$ hexagons (Gan et al., 2009). The non-classical fullerenes are defined as cages containing a number of pentagons different from 12 and one or more defects such as heptagons, squares (Gan et al., 2009; Tang et al., 2011; Xiu-Fang et al., 2008) (cf. Figure 1). The open cage group include cage clusters that contain defects with more than seven atom cycles.

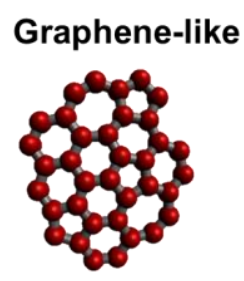

(a)

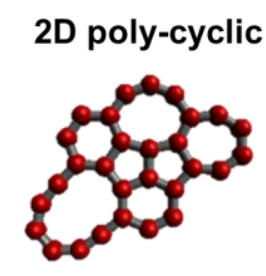

(b)

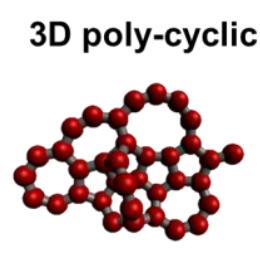

(c)

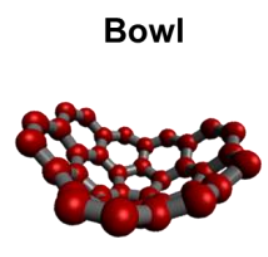

(d)

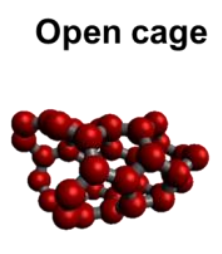

(e)

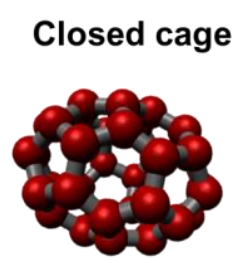

(f)

Figure 1: The six structural groups taken into account in the present statistical study.

It is worthy to mention that the classification adopted in this work implies that the large number of classical and non-classical fullerenes that exist for large size clusters, such as those reported in reference (Tang et al., 2011) were lumped under the same isomer class, i.e., closed cage group. Similarly, the numerous 2D-graphenelike isomers such as those reported in reference (Kosimov et al., 2010) were lumped in the 2D-Graphene-like class. Such a simplified six-class description enables limiting the number of trajectory simulations since we are seeking the statistical occurrence of only six states. Of course, the drawback of such a choice is that we do not get detailed information with respect to the abundance of the large number of isomers that belong to the same class, such as the classical fullerene isomers that belong to the cage class.

A typical temperature time-variation used in the present CCA MD-simulations is shown in Figure 2. It is characterized by (i) a heating phase, with a characteristic time $\tau_{\mathrm{h}}$ and a heating rate $R_{H}$, that starts with a randomly distributed $\mathrm{n}$ carbon atoms at a temperature $\mathrm{T}_{\min }$ and ends up at a temperature $\mathrm{T}_{\max }$, (ii) a constant temperature regime (CTR) at $\mathrm{T}_{\max }$ with a duration $\tau_{C T R}$ during which the carbon structure obtained at the end of the heating phase further relaxes and (iii) a cooling phase with a duration $\tau_{c}$ and a cooling rate $R_{c}$ during which the temperature of the carbon structure decreases down to $\mathrm{T}_{\min }$.

As mentioned previously, when produced under non equilibrium conditions, carbon nanostructures can experience temperature fluctuations with pic values much above 1000-2000 K (Prasanna et al., 2019). 
Therefore, in order to investigate the abundance of the clusters isomers over all the temperature range that may be reached while they are produced, CCA MD-simulations were performed for three values of $\mathrm{T}_{\max }: 2000 \mathrm{~K}$, $3000 \mathrm{~K}$ and $4000 \mathrm{~K}$.

Results of the study performed in reference (Gao et al., 2007a) where the authors make use of simulated annealing MD-simulation in order to determine the most stable isomer of $\mathrm{C}_{36}$ showed that a typical value of $100 \mathrm{~ns}$ relaxation time is needed to insure a relaxation phase during which the cluster explore a large enough domain of the potential hypersurface so as to visit a large number of local minima. Therefore, a base value of $100 \mathrm{~ns}$ was set for the heating time $\tau_{\mathrm{h}}$ and a parametric study was performed in order to investigate the sensitivity of the CCA MD-simulations in terms of isomer abundance to the adopted value of $\tau_{\mathrm{h}}$. We choose the same base value of $100 \mathrm{~ns}$ for the cooling time $\tau_{\mathrm{c}}$.

The present study is carried out with either canonical or microcanonical constant $(\mathrm{N}, \mathrm{V}, \mathrm{T})$ and $(\mathrm{N}, \mathrm{V}, \mathrm{E})$ ensembles. Our CCA simulation represents a thermodynamic transformation of $\mathrm{n}$ carbon atom to a single $\mathrm{C}_{\mathrm{n}}$ cluster in vacuum. For a given cluster size, the size of the simulation box implicitly determines the initial density of the carbon cloud, and may influence the simulation results. A $\left(100 \mathrm{~A}^{\circ}\right)^{3}$ base value, of the same order of those with those used in the literature for carbon cluster condensation (Gao et al., 2007b; Lichtenegger and von der Linden, 2009; Yamaguchi and Maruyama, 1998), was used for the size of the simulation box and the sensitivity of the simulation to this parameter was analyzed by comparing with the results obtained when using a larger simulation box.

It is also worthy to mention that the CCA process is considered under vacuum in the present study. One may also consider similar transformation but under constant and finite pressure. However investigating the pressure effect calls for the use a different thermodynamic ensemble, i.e., the (N,P,T) ensemble, that requires a suitable Hamiltonian formulation and specific simulation approach (Andersen, 1980; Martyna et al., 1994; Uline and Corti, 2013). This is out of the scope of the present work.

The statistical abundance of the different isomers of a given $\mathrm{C}_{\mathrm{n}}$ cluster is determined by performing CCA MDsimulations that start with $\mathrm{n}$ carbon atoms that are randomly positioned in space at a temperature of $0.1 \mathrm{mK}$. These atoms are then submitted to the three-phase combined condensation-annealing process described previously in a $100 \times 100 \times 100 \mathrm{~A}^{\circ 3}$ simulation box with periodical boundary conditions. The velocity Verlet algorithm is used to integrate Newton's equations of motion for the $\mathrm{n}$ carbon atoms inside the simulation box. The integration time step was set to $0.1 \mathrm{fs}$. This value ensures stable dynamics trajectories and enables capturing the chemical bonding process and conserving the total energy in one time iteration in the micro-canonical ensemble NVE. Since we are mainly interested in the statistical abundance independently of the environment, the temperature of the system is next adjusted using Berendsen thermostat with a thermal relaxation time of 1 


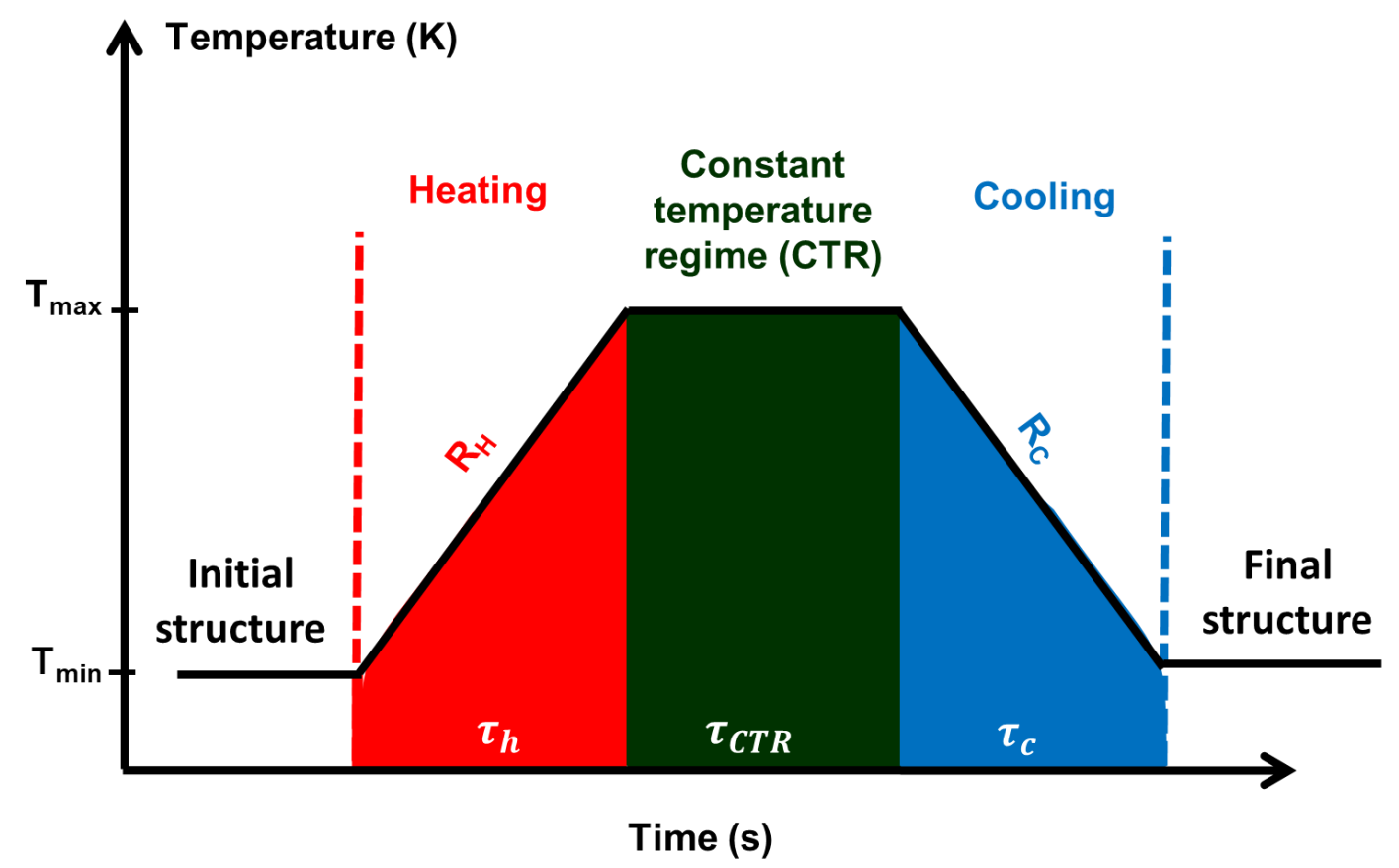

Figure 2: Diagram representing the three phases of CCA method.

\section{Results and discussion.}

\subsection{Setup of the simulation methodology}

The simulation procedure defined in the previous section involves several parameters, the values of which may affect the simulation results. We need first to validate the parameters involved in the CCA cycles.

The first point that we investigated in order to setup our simulation methodology is related to the number of trajectory simulations needed to reach statistically meaningful abundance of the six clusters group. Once the number of simulations has been chosen, we investigated how the determined statistical abundance is affected by the durations of the heating, constant temperature regime and cooling phases of the CCA cycle.

\section{1. a. Number of trajectory simulations}

A first set of simulations was carried out, with 36 free atoms submitted to a CCA cycle with a maximum temperature of $3000 \mathrm{~K}$ and total duration of $300 \mathrm{~ns}$. Two typical illustrations of the evolution of 36 free carbon atoms to either 2D-graphene-like cluster or cage-like $\mathrm{C}_{36}$ cluster is shown in Figure $3 \mathrm{a}$ and $3 \mathrm{~b}$, respectively. Some of the features seen in these two figures were observed in all the simulations performed in this work, i.e., around 1500 simulations. As a matter of fact, we systematically observed that : (i) the early stage of the 
condensation process always results in the formation of $2 \mathrm{D}$ or $3 \mathrm{D}$ polycyclic clusters, (ii) the structural group that results from a given simulation is always obtained before the end of the CTR phase that last in our case for $100 \mathrm{~ns}$ and (iii) although the structural group of the cluster does not change during the cooling phase, the annealing could be very effective and results in further stabilization of the cluster inside the same functional group. With respect to this last point, we observed for example in some simulations that a $100 \mathrm{~ns}$ cooling phase resulted in the formation of the more stable isomer, e.g. $\mathrm{D}_{6 \mathrm{H}}$ classical cluster in the case of $\mathrm{C}_{36}$ (Gao et al., 2007a).

We performed 100 CCA MD-simulations and followed the averaged statistical abundance and corresponding standard deviation as function of the number of simulations for 4 clusters, $\mathrm{C}_{21}, \mathrm{C}_{29}, \mathrm{C}_{36}$ and $\mathrm{C}_{50}$. The obtained results are illustrated in figure Figure 4 in the case of $\mathrm{C}_{21}(\mathrm{a})$ and $\mathrm{C}_{36}(\mathrm{~b})$. We also show in Table 1 the mean the abundances calculated by averaging the results obtained between the $75^{\text {th }}$ and the $100^{\text {th }}$ simulations, along with the standard deviations from this means. We clearly see from Figure 4 that a statistically representative abundance value is obtained after only 25 simulations. Table 1 shows that the standard deviation calculated for the 25 last simulations is around $0.5 \%$, which further confirm the validity of our statistical representation. Therefore, we decided to perform for each cluster size $100 \mathrm{CCA}$ MD-simulations and to determine the statistical abundance and the corresponding standard deviation for the different structural groups as described above. 


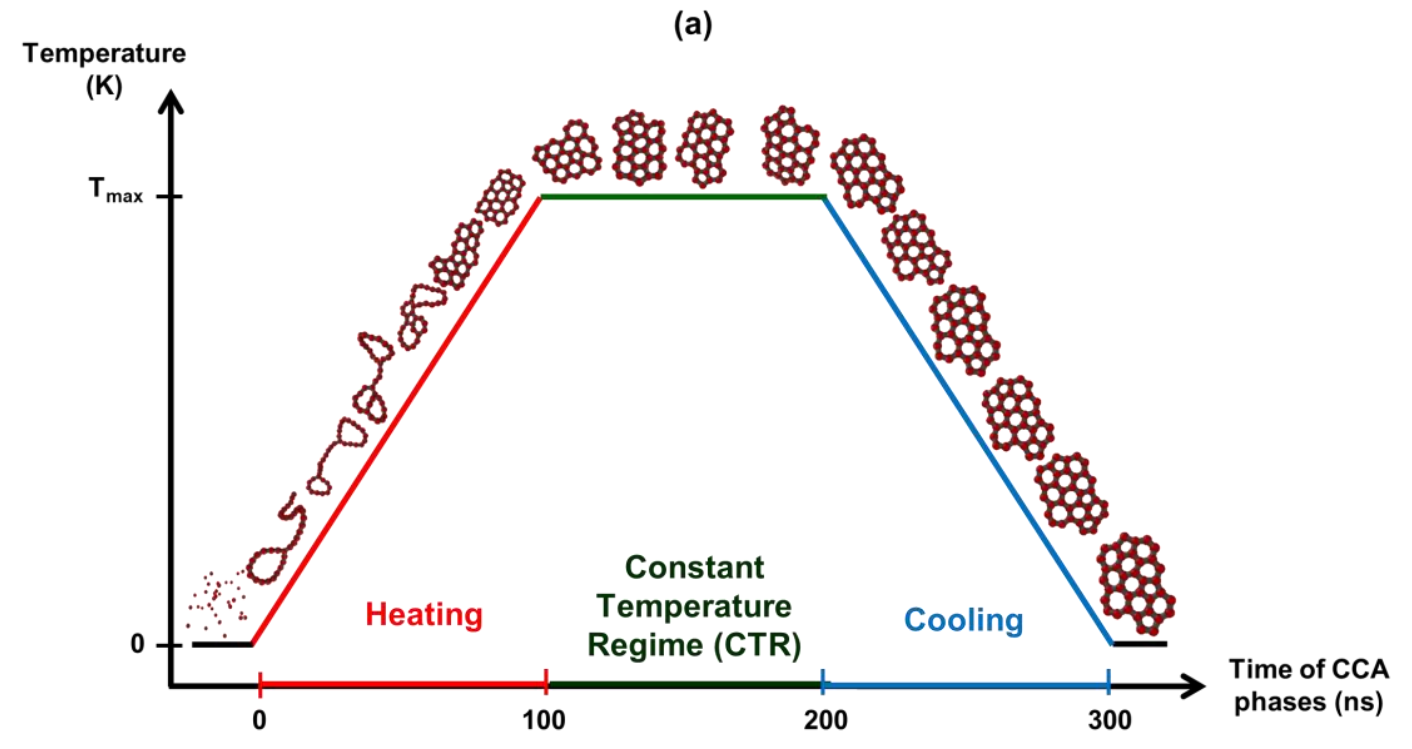

(b)

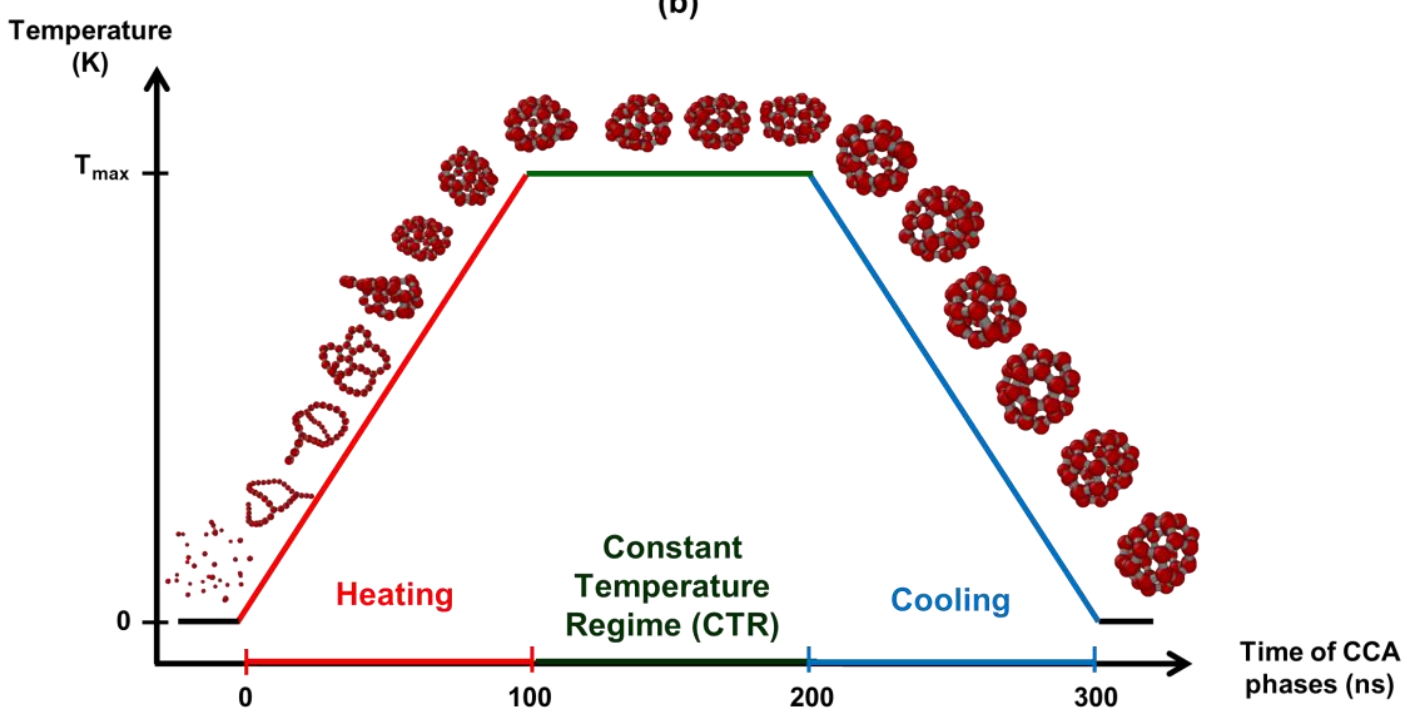

Figure 3: Illustration of two typical CCA simulations performed on two random spatial distributions of 36 free carbon clusters and that end up by the formation of a 2D-graphene-like (a) and a closed cage-like (b) $\mathrm{C}_{36}$ clusters. The simulation is performed for $\mathrm{T}_{\max }=3000 \mathrm{~K}$, heating and cooling times of $100 \mathrm{~ns}$ and a CTR phase of $100 \mathrm{~ns}$. 

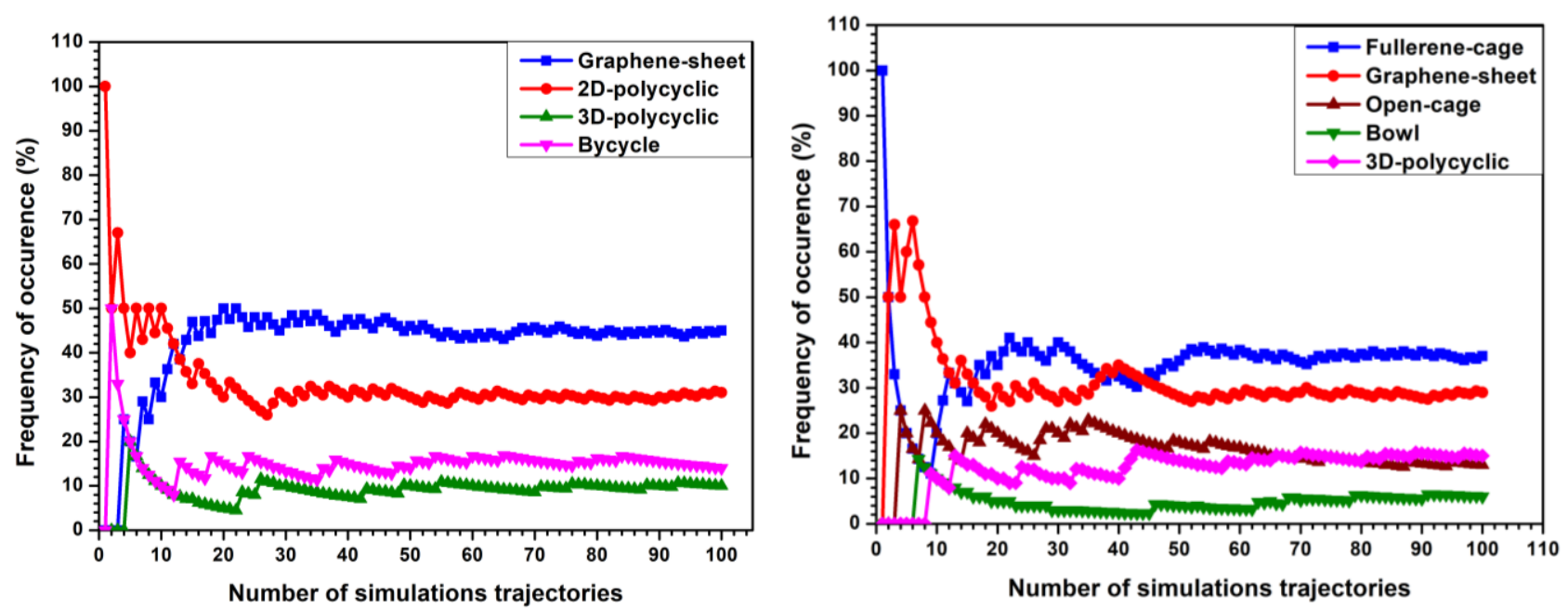

Figure 4: Evolution of the averaged abundances of the difference classes as function of the number of simulations for $\mathrm{C}_{21}$ (a) and $\mathrm{C}_{36}$ (b). A statistically representative values are obtained after only 25-30 simulations for $\mathrm{C}_{21}$ and 50 simulations for $\mathrm{C}_{36}$.

Table 1 : Mean-values and standard deviation of the statistical abundances for $\mathrm{C}_{21}$ and $\mathrm{C}_{36}$ obtained by averaging the results obtained between the $75^{\text {th }}$ and $100^{\text {th }}$ simulations.

\begin{tabular}{|l|c|c|c|c|}
\hline & \multicolumn{2}{|c|}{$\mathrm{C}_{21}$} & \multicolumn{2}{c|}{$\mathrm{C}_{36}$} \\
\hline Fullerène & Average (\%) & $\begin{array}{c}\text { Standard } \\
\text { deviation (\%) }\end{array}$ & Average (\%) & $\begin{array}{c}\text { Standard } \\
\text { deviation (\%) }\end{array}$ \\
\hline Graphene & - & - & 37 & 0.5 \\
\hline Open cage & - & 0.4 & 29 & 0.5 \\
\hline Bowl & - & - & 13 & 0.5 \\
\hline 3D polycyclic & 10 & 0.4 & 15 & 0.4 \\
\hline 2D polycyclis & 30 & 0.5 & - & - \\
\hline Bycyclic & 15 & 0.7 & - & - \\
\hline
\end{tabular}

\section{1. b. Constant temperature regime (CTR) duration}

The first characteristic parameter for the CCA transformation is the waiting phase duration. Maruyama et Yamaguchi ( Maruyama and Yamaguchi, 1998) showed that the relaxation of cage-like carbon cluster to stable 
fullerene isomers in simulated-annealing requires relaxation times of the order of 100 ns. Although, our simulations last for more than $200 \mathrm{~ns}$, a fraction of this time is devoted to the heating and cooling processes and a question arises with respect to the fraction of time that should be devoted to the CTR phase. A short timeduration of the CTR phase would enable reducing the computation time provided the estimated statistical abundance of the six structural groups is not affected. On the other hand, a long CTR phase duration requires a long simulation time but would enable exploring much larger domain of the potential hypersurface at the annealing temperature, which makes it possible to reach a larger number of locally stable configurations.

We therefore performed CCA simulations with CTR phase durations of $100 \mathrm{ps}$ and $100 \mathrm{~ns}$ for annealing temperatures of $2000 \mathrm{~K}, 3000 \mathrm{~K}$ and $4000 \mathrm{~K}$. The results are shown in Figure 5 for C36. It appears first that the two CTR phase durations yields similar results in many cases. For instance, for both CTR phase durations, closed-cage and graphene-like groups represent 35-40\% and 30\% of the clusters for annealing temperature of $3000 \mathrm{~K}$. Both CTR phase durations yield little more than $40 \%$ abundance for the dominant open-cage group, a very small occurrence for bowl-like isomers and no closed cage isomers at $2000 \mathrm{~K}$. A larger abundance of the 2D-graphene-like and smaller abundance of the 3D-polycyclic group are however obtained when a longer CTR phase duration is used. Note however that the total abundances of the two groups obtained for the two CTR phase values are similar, which shows that the main effect of an increased CTR phase duration at $2000 \mathrm{~K}$ is a further transformation of the 3D-polycyclic structures to 2D-graphene-like structures. The sensitivity of the predicted abundance to the CTR phase duration is much more pronounced at $4000 \mathrm{~K}$. It appears indeed that a longer CTR phase at $4000 \mathrm{~K}$ tends to significantly favor the formation of 2D and 3D polycyclic clusters, while reducing the abundance of 2D-graphene-like and open cage clusters. These results show that at least $100 \mathrm{~ns}$ CTR phase duration is required for the estimation of the statistical abundances over a wide range of temperature.

Figure 5 presents the details of the energy spectra of the isomers within the different structural groups obtained for CTR phase durations of $100 \mathrm{~ns}$ (a) and $100 \mathrm{ps}$ (b) at $3000 \mathrm{~K}$. It appears that although the two sets of simulation yield very similar statistical abundance of the different structural groups, the energy of the most stable isomers obtained with the two CTR phase durations are significantly different for some structural groups. The simulations performed with 100 ps CTR phase duration systematically yield more stable structure inside each structural class. Following Gao et al., this result may be possibly explained by the fact that a long time duration CTR phase at $3000 \mathrm{~K}$ involves significant entropic component of Helmoltz free energy, thus stabilizing structure with higher potential energy, i.e., the most likely structure is the one with the lowest Helmoltz free energy which is not necessary the one that shows the lowest potential energy. In any case, the statistical abundance of the different structural groups is not affected when the CTR phase is decreased from $100 \mathrm{~ns}$ to $100 \mathrm{ps}$. 

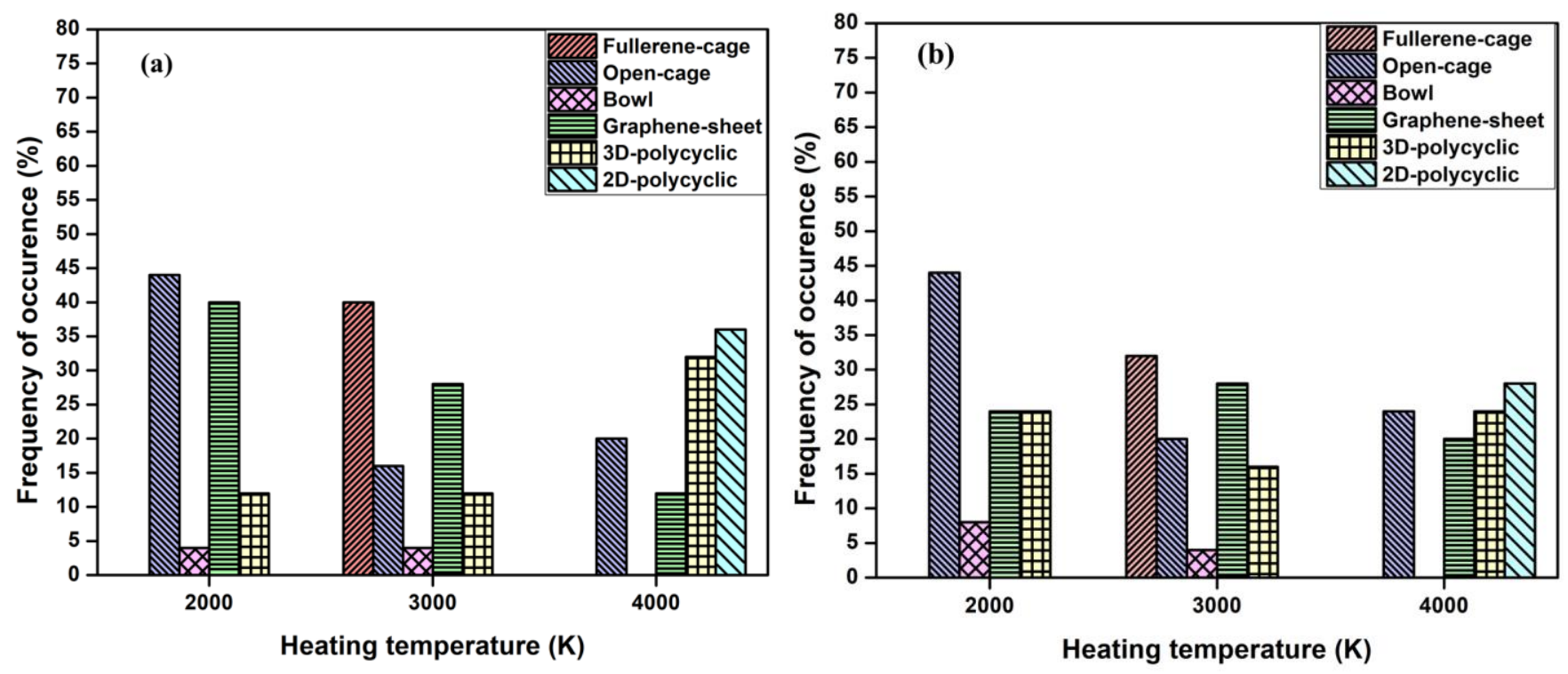

Figure 5: Statistical abundance of the six structural groups determined using $100 \mathrm{~ns}$ (a) and 100 ps (b) CTR phase durations for maximum temperature values of $2000 \mathrm{~K}, 3000 \mathrm{~K}$ and $4000 \mathrm{~K}$. 

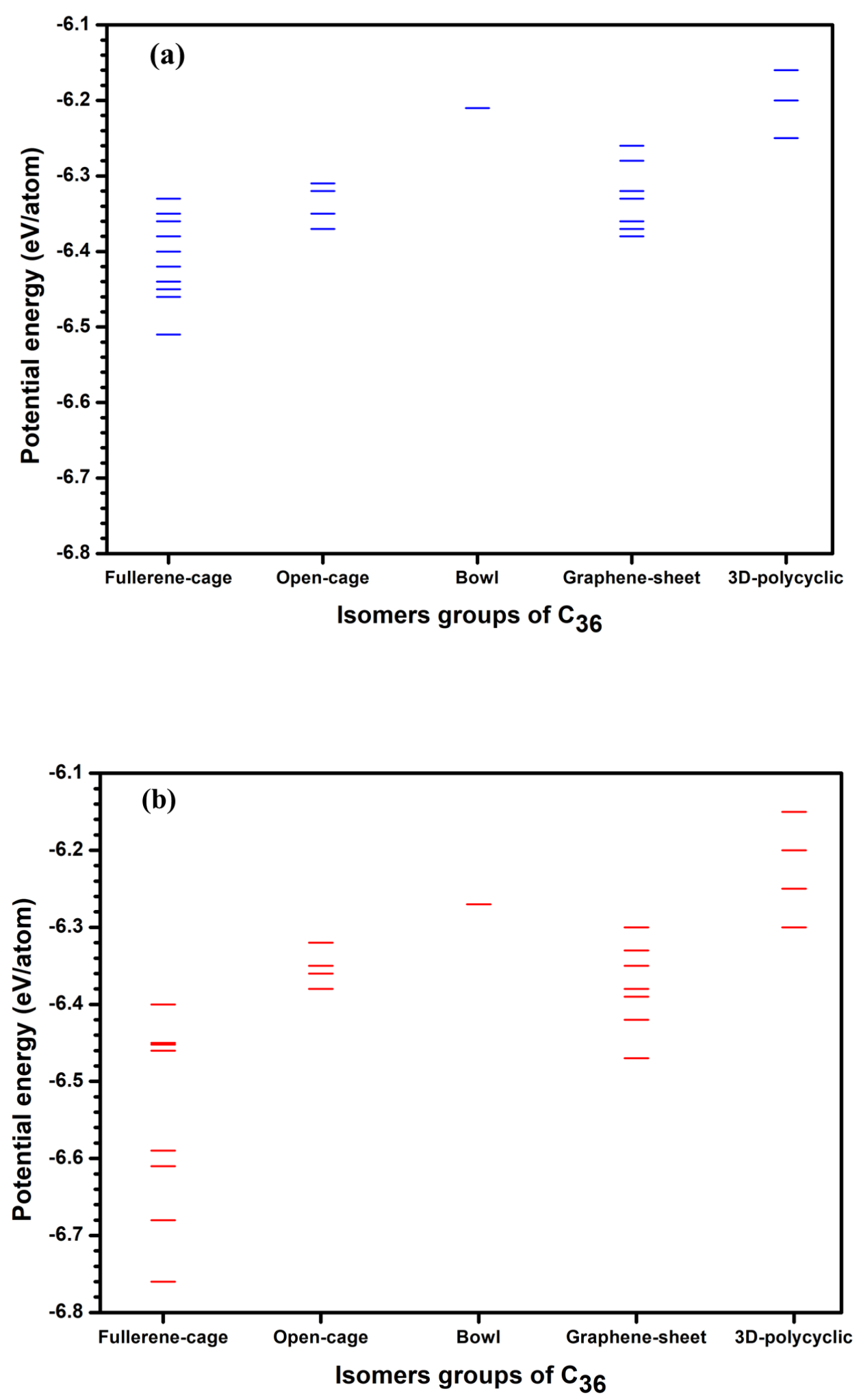

Figure 6: Energy spectrum of the clusters obtained for the different structural groups for combined condensation/annealing performed at a maximum temperature of $3000 \mathrm{~K}$ and CTR phase durations of $100 \mathrm{~ns}$ (a) and $100 \mathrm{ps}(\mathrm{b})$. 


\section{1. c. Heating phase duration:}

We used $100 \mathrm{~ns}$ duration, i.e., $3 \times 10^{10} \mathrm{~K} \cdot \mathrm{s}^{-1}$ heating rate, for the heating phase where both condensation and heating take place. This would result, with a CTR phase of $100 \mathrm{~ns}$, in a $200 \mathrm{~ns}$ relaxation, before the cluster is submitted to the cooling phase. We have observed in all our simulations that the final structural group of the cluster is obtained for typical values of the relaxation time between $100 \mathrm{~ns}$ and $200 \mathrm{~ns}$, which is in agreement with the conclusion of reference (Gao et al., 2007a; Maruyama and Yamaguchi, 1998), that indicates that relaxation time above $100 \mathrm{~ns}$ is required for kinetic equilibrium. For longer relaxation times, the clusters remain in the same structural group although they may experience a slight structural change and stabilization. This means that with a $100 \mathrm{~ns}$ CTR phase, a heating phase longer than $100 \mathrm{~ns}$ is not necessary. Nevertheless, in order to see whether it is possible to reduce the simulation time and investigate the effect of the adopted heating time values on the predicted abundances, we performed simulations with shorter heating time, i.e., $10 \mathrm{~ns}$ and $1 \mathrm{~ns}$ (cf. Figure 7). It appears that for very short rise time, i.e., $1 \mathrm{~ns}\left(3 \times 10^{12} \mathrm{~K} \cdot \mathrm{s}^{-1}\right.$ heating rate), the condensation is never complete and results in a significant amount of small fragments (cf. Figure 7). For 10 ns heating time, i.e., $3 \times 10^{11} \mathrm{~K} . \mathrm{s}^{-1}$, the condensation is completed but the annealing does not allow a full relaxation and result in a large fraction of the less stable 2D-graphene like clusters (cf. Figure 7). It appears that combined condensation-annealing with $100 \mathrm{~ns}$ heating time tends to result in a large abundance of the most stable group, i.e., closed cage-like group, and that for all the simulation performed with this value of the heating time, the final structural group is reached before the end of the CTR phase. We therefore adopt a value of $100 \mathrm{~ns}$ in the rest of the study.

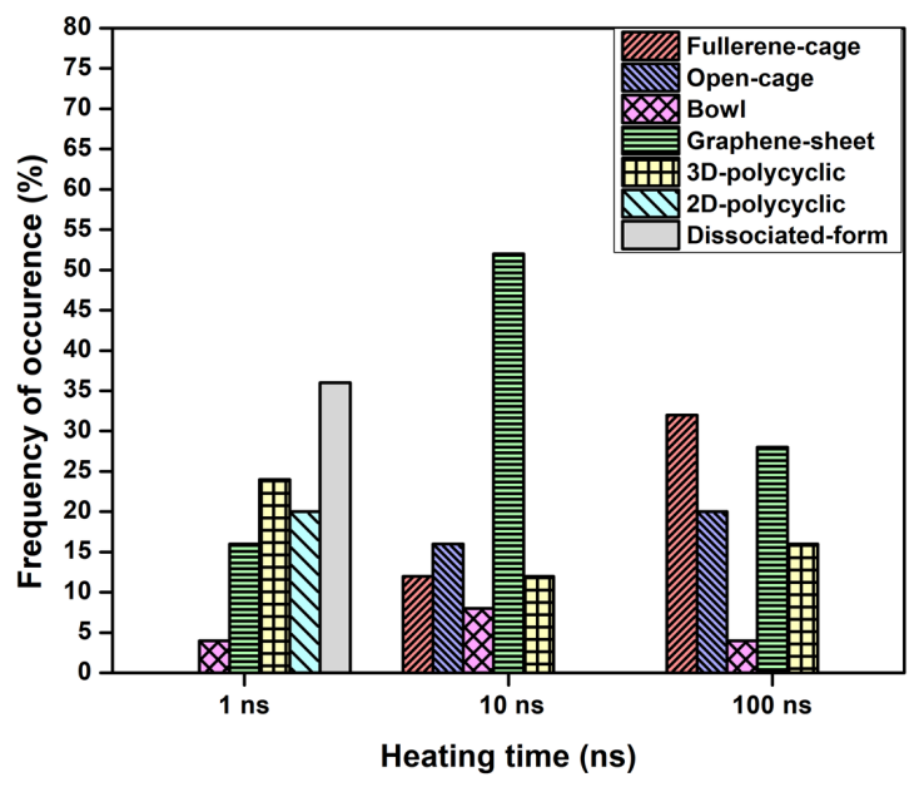

Figure 7 : Effect of heating time on the statistical abundance of the different structural groups for $\mathrm{C}_{36}$ carbon clusters, and evolution of the cluster configuration during the CCA cycle for different heating time at a maximum temperature of $3000 \mathrm{~K}$. 


\section{1. d. Cooling phase duration}

As mentioned previously, when using a value of $100 \mathrm{~ns}$ for the heating and CTR times, in the combined condensation-annealing transformation, the structural group of the cluster is defined at the end of the CTR phase. For instance, Figure 8 shows that $10 \mathrm{~ns}$ and $100 \mathrm{~ns}$ cooling phase durations yield the same statistical abundance for the dominant structural groups, i.e., 35\% for the closed cage group, 30\% for the 2D-graphene like group, $20 \%$ for the open cage group. The same abundance values are also obtained for the very minor bowl-like structural group. The only difference is observed on the $2 \mathrm{D}$ - and 3D-polycyclic groups. The two cooling phase durations yield the same value for the total abundance of the 2D-and 3D-polycyclic groups. However, longer cooling phase durations yield an enhanced conversion of the 2D-polycyclic to 3D-polycyclic isomers, and therefore to larger 3D-polycyclic abundance and smaller 2D-polycyclic abundance. These results show that $10 \mathrm{~ns}$ cooling phase duration would be enough for the determination of the statistical abundance of the major structural groups. In fact, using cooling phase duration as long as $100 \mathrm{~ns}$ makes it possible to explore a larger region of the potential surface, thus enabling reaching more stable isomers inside a given structural group. In fact, even 1ns duration of the cooling phase results in a statistical abundance that is not very different from those obtained for 10 and $100 \mathrm{~ns}$ for a CTR phase temperature of $3000 \mathrm{~K}$. The fractions of open cage and bowl-like isomers are indeed only slightly larger for such short cooling duration. The abundances of all cagelike isomers (both open and closed), graphene-like and all polycyclic isomers (both 3D and 2D) are moreover almost identical to those obtained for $10 \mathrm{~ns}$ and $100 \mathrm{~ns}$.

Figure 9 shows for example the energy spectrum of the isomers belonging to the different structural groups. It appears that the CCA simulations conducted with cooling phase duration of $100 \mathrm{~ns}$ generally yield more stable isomers for all the structural groups. For instance, the energy of the cage-like isomers obtained for $100 \mathrm{~ns}$ cooling phase duration is approximately $-6.8 \mathrm{eV}$, while the cage-like isomers obtained for $1 \mathrm{~ns}$ and $10 \mathrm{~ns}$ cooling time are much less stable with energy values of $-6.5 \mathrm{eV}$ and $-6.58 \mathrm{eV}$. It is worthy to mention at this stage that the energy value of the most stable cage-like isomers found with a short duration CTR phase and $100 \mathrm{~ns}$ cooling phase is comparable to the value ofD6hfullerene structure reported by Gao et al. (Gao et al., 2007a).

It appears therefore that although the obtained isomer abundances are not sensitive to the cooling phase duration, longer cooling time seems to enable exploring a much a larger domain of the potential hypersurface, thus enhancing the chance, for a given cluster class, to detect more stable isomer. We therefore chose to use CCA MD-simulations with 100 ns cooling-time duration. 


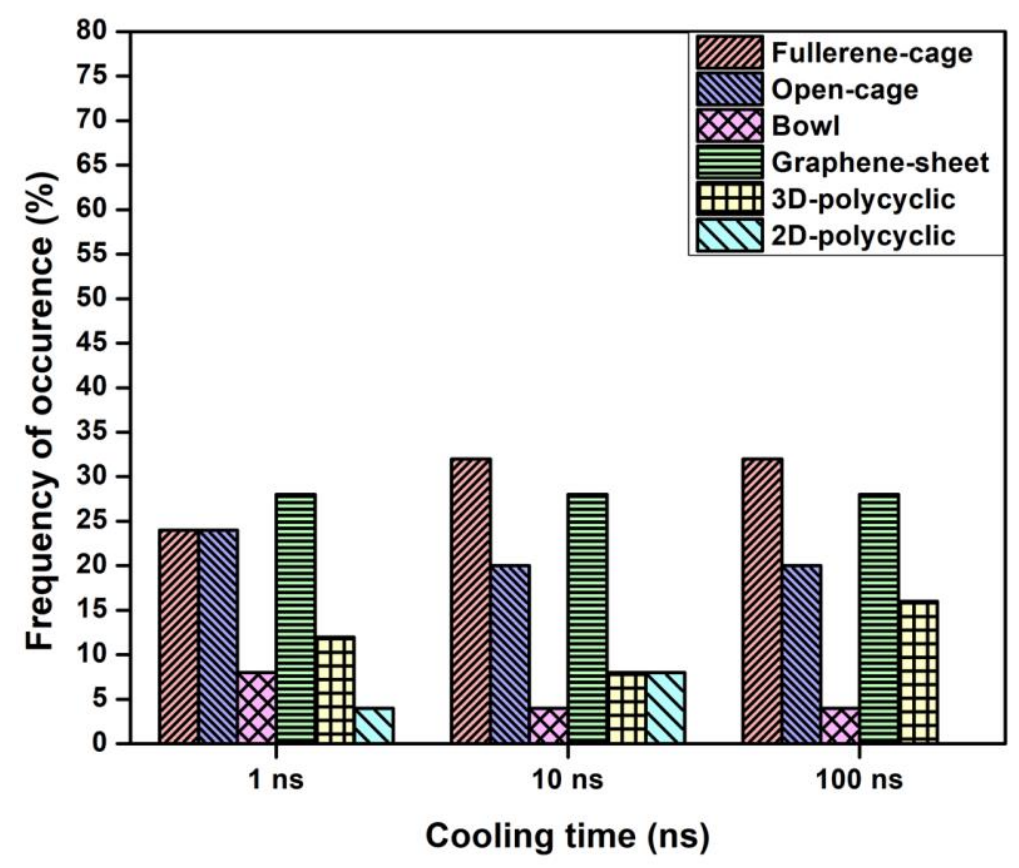

Figure 8: Statistical structural group abundances for three different values of the cooling phase duration. Energy spectra of the cluster structures obtained with $1 \mathrm{~ns}, 10$ and $100 \mathrm{~ns}$ cooling time. The CTR phase temperature is $3000 \mathrm{~K}$. 

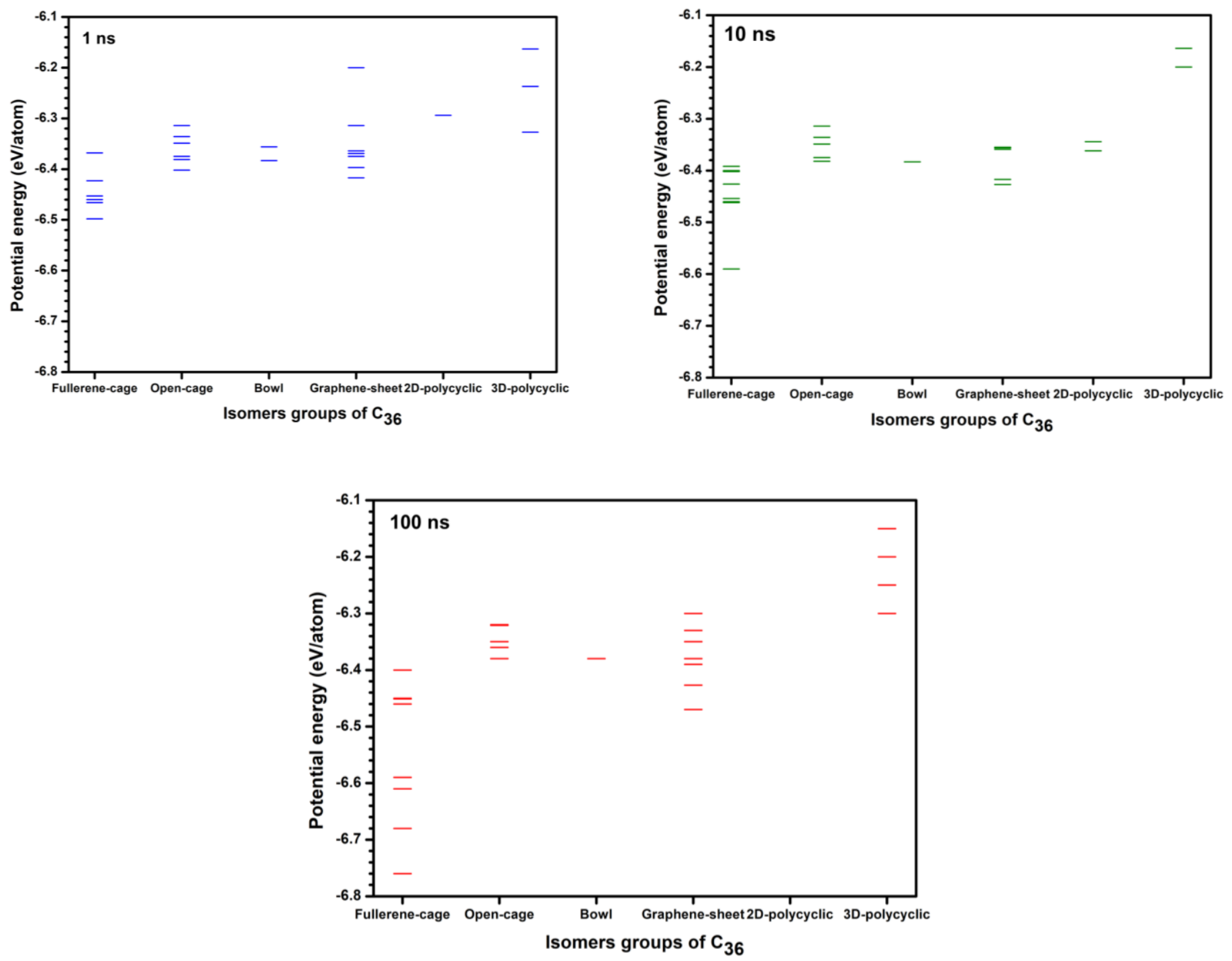

Figure 9: Energy spectra of the isomers obtained for the different structural groups from CCA MDsimulations with $1 \mathrm{~ns}, 10 \mathrm{~ns}$ and $100 \mathrm{~ns}$ duration of the cooling phase. 


\section{1.e. Effect of the size of the simulation box}

The size of the simulation box used is one of the major parameters in CCSA MD-simulation. This size determines the initial density of the carbon cloud and may affect the simulation results. Our first choice of $\left(100 \mathrm{~A}^{\circ}\right)^{3}$ simulation box is motivated by the fact that most of the works published on the simulation of carbon cluster condensation were performed with the same order of the simulation box size (Gao et al., 2007b; Lichtenegger and von der Linden, 2009; Yamaguchi and Maruyama, 1998). It is worthy to mention here that whatever the simulation box size, the simulation with the periodic boundary conditions used here corresponds to a thermodynamic transformation where the carbon atoms of the initial 'gaseous' cloud interact with each other until they end up in a single cluster. The size of the simulation box should essentially determine the time required for such a "condensation". Nevertheless, in order to investigate the effect of the size of the simulation box on the simulation results we performed simulations with a $\left(200 \mathrm{~A}^{\circ}\right)^{3}$ simulation box. In figure 9new we show the evolution of the average statistical abundances of fullerene and graphene isomers obtained with $\left(100 \mathrm{~A}^{\circ}\right)^{3}$ and $\left(200 \mathrm{~A}^{\circ}\right)^{3}$ for $\mathrm{C}_{36}$. We can see that the two simulation box sizes give almost the same results for both isomers. More precisely, the average abundance of graphene calculated for the last 25 simulation is 30 with standard deviation of 2.3 and 28 with a standard deviation of 1.7 for the $\left(100 \mathrm{~A}^{\circ}\right)^{3}$ and $\left(200 \mathrm{~A}^{\circ}\right)^{3}$, respectively. Similarly, the average abundance of fullerene calculated for the last 25 simulation is 34 with standard deviation of 2.8 and 32 with a standard deviation of 1.3 for the $\left(100 \mathrm{~A}^{\circ}\right)^{3}$ and $\left(200 \mathrm{~A}^{\circ}\right)^{3}$, respectively.
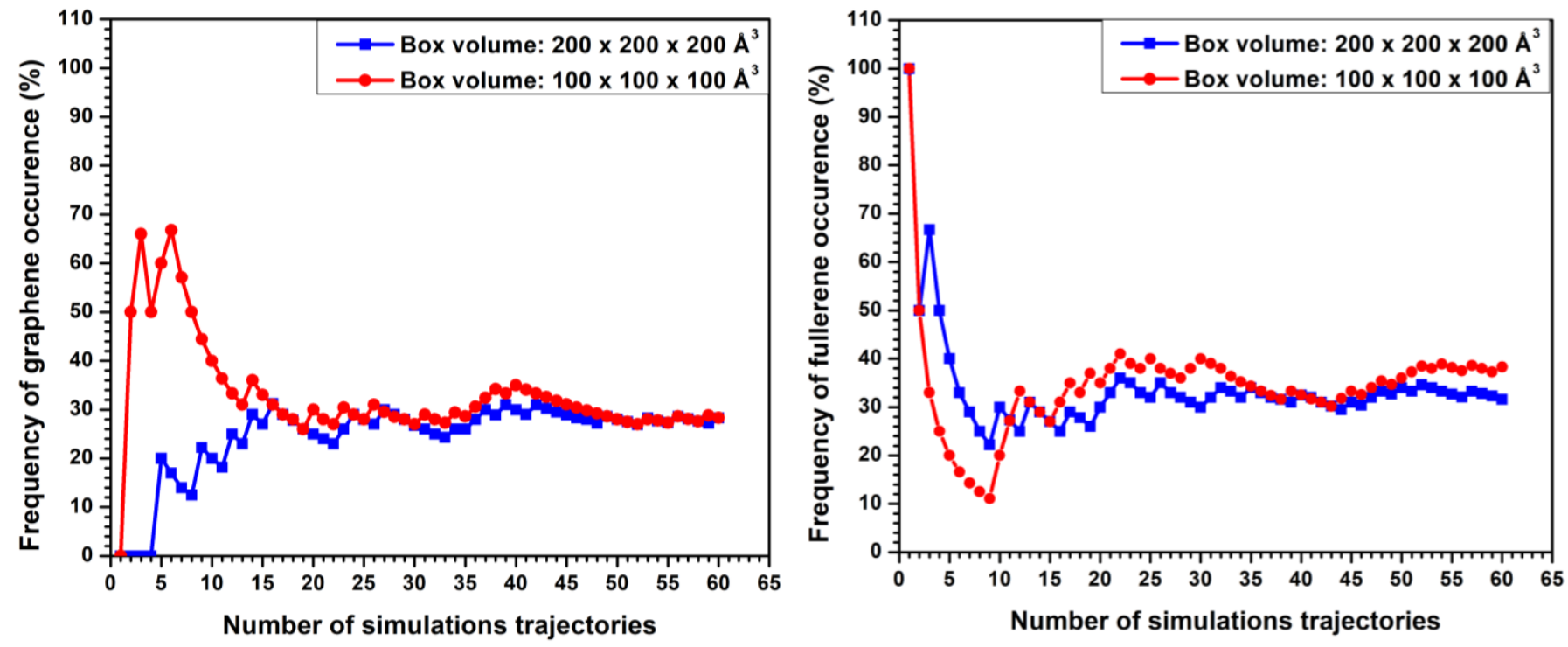

Figure 10: Evolution of averaged statistical abundances of $\mathrm{C}_{36}$ fullerene and graphene like isomers as function of the simulation number for $\left(100 \mathrm{~A}^{\circ}\right)^{3}$ and $\left(200 \mathrm{~A}^{\circ}\right)^{3}$. 


\section{1. f. Adopted simulation parameters}

The analysis above shows that the best parameters set for the determination of the abundance of the structural groups by CCA MD-simulations are a CTR phase duration of $100 \mathrm{~ns}$ and a heating time of $100 \mathrm{~ns}$. Although it is not as much necessary, we choose to use a cooling time of 100 ns, i.e., 100 ns/100 ns/100 ns CCA MDsimulations. Besides, we also conducted simulation with shorter CTR phase duration, i.e., 100 ps, since it appeared that this makes it possible to reach significantly lower energy structures. In any case the minimum energy clusters obtained with 100 ps CTR phase will be compared to those published by other authors in order to analyze the capability of this $100 \mathrm{~ns} / 0.1 \mathrm{~ns} / 100 \mathrm{~ns}$ CCA simulation to reach the most stable cluster structures.

\subsection{Analysis of the cluster structure obtained by CCA MD-simulations}

In this section, we first show typical cluster geometries as function of cluster size as obtained using $100 \mathrm{~ns} / 100$ ps/100 ns CCA MD -simulations. Then we give the abundance of the different structural groups for 4 cluster sizes, i.e., $\mathrm{C}_{22}, \mathrm{C}_{29}, \mathrm{C}_{36}$ and $\mathrm{C}_{50}$, as obtained from $100 \mathrm{~ns} / 100 \mathrm{~ns} / 100 \mathrm{~ns}$ CCA simulations. We end up the section by comparing the energy of the most stable structures obtained from CCA simulations and other approaches published in the literature.

\section{2. a. Typical cluster geometries obtained from CCA MD-simulations for $C_{n}(2 \leq n \leq 54)$ clusters}

In Figure 11 we report energy diagram showing the structures obtained by CCA MD-simulations for different cluster sizes. Linear structures are observed for cluster sizes in the range $n=3-10$. Mono-ring structures are obtained over a large size domain, for $n=6-18$. 2D double-rings structures are observed for cluster sizes in the range $n=10-23$. Three-ring structures are obtained for cluster size between $n=15$ and $n=22$. 2D- and 3Dpolycyclic structures are obtained for cluster size in the ranges $n=16-35$ and $n=20-40$, respectively. 2D graphene-like structures with typical pentagonal and/or heptagonal edge-defects are observed for cluster size above $n=20$. Open cage is observed for cluster size above $n=25$, while closed cage are obtained only for $n \geq$ 30.

The number of isomers varies significantly as function of the cluster size. There is only a single linear structure for small clusters with $\mathrm{n} \leq 5$, while two isomers are obtained for clusters in the ranges $\mathrm{n}=6-9$ (linear and monorings) and 11-13 (mono-rings and double-rings). Three isomers are obtained for cluster size of n=10 (one-, two- and three-ring), $n=15$ (one-, two- and three-ring) and $n \geq 40$ (open-cage, closed-cage and graphene). More than 3 and up to 5 isomers are obtained for the intermediate size clusters $n=16-39$. 


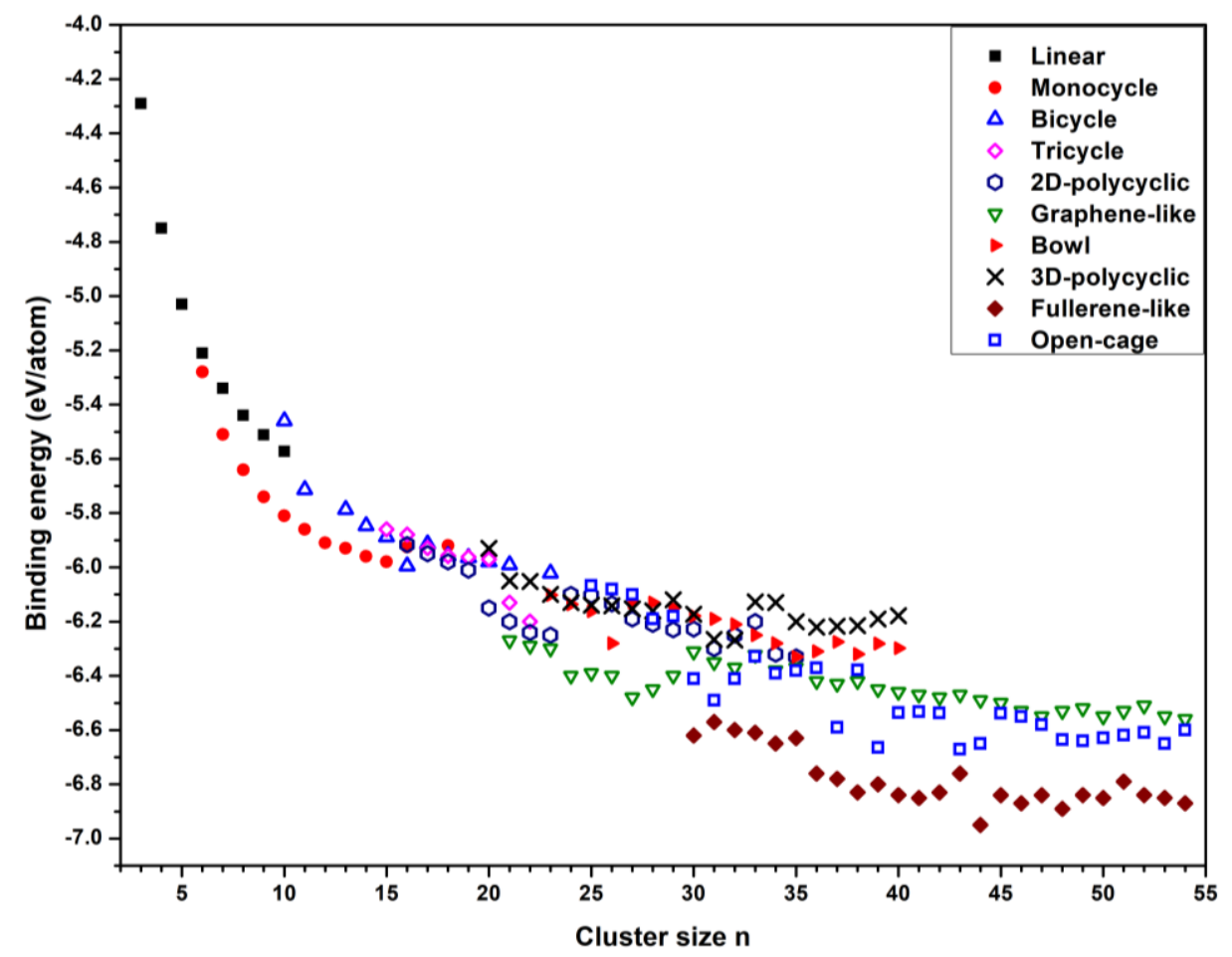

Figure 11: Energy diagram showing the structures obtained for each size. The simulations are performed for $\mathrm{T}_{\max }=3000 \mathrm{~K}$, heating and cooling times of $100 \mathrm{~ns}$ (i.e., $3 \times 10^{10} \mathrm{~K} \cdot \mathrm{s}^{-1}$ heating and cooling rate) and a CTR phase of 100 ps.

\section{2. b. Structural group abundance for $C_{21}, C_{29}, C_{36}$ and $C_{50}$ clusters}

The abundance of the structural groups was determined as described in section 3.1.1 for an annealing temperature of $3000 \mathrm{~K}$ except in the case of $\mathrm{C}_{50}$ for which we obtained statistically significant results after only 80 simulations. The results are shown in Figure 12. The $\mathrm{C}_{21}$ cluster is dominated by the graphene-like group (45\%) and the 2D-polycyclic structures (45\%), among which $14 \%$ are two-ring clusters. No open- or closecage structures were obtained for this cluster. With $39 \%$ abundance, 2D-graphene-like structure is still dominant in the case of the $\mathrm{C}_{29}$ cluster that also shows significant fractions of the 2D- (27\%) and 3D (20\%) polycyclic structures. A small fraction of closed (8\%) and open (6\%) cage-like isomers was also observed in this case. As for the $\mathrm{C}_{36}$ cluster, closed-cage group and 2D-graphene-like isomers are dominant with $37 \%$ and 29\% abundance. Open cage and 3D-polycyclic structural groups also show significant abundances, i.e., 13\% and $15 \%$. Large clusters such as $\mathrm{C}_{50}$ are essentially dominated by cage-like structures, mainly closed cage that represents $76 \%$ of the population. The rest of the clusters, $11 \%$, show a graphene-like structure.

In fact, Graphene-like and 2D-polycyclic structures dominate not only $\mathrm{C}_{21}$ and $\mathrm{C}_{29}$ but all the clusters in the size range 17-29. The fractions of these isomers tend to decrease with the cluster size. Nevertheless, graphene- 
like structures are still obtained for $\mathrm{C}_{50}$. Although they are observed for small and intermediate size clusters, the 3D-polycyclic structures are only significant for the $\mathrm{C}_{29}$. They are not observed for the large $\mathrm{C}_{50}$ cluster. The abundance of open- and closed-cage structure increases with the cluster size. Bowl structure was only obtained for $\mathrm{C}_{36}$. Its abundance remains however very small at the considered temperature.

(a)

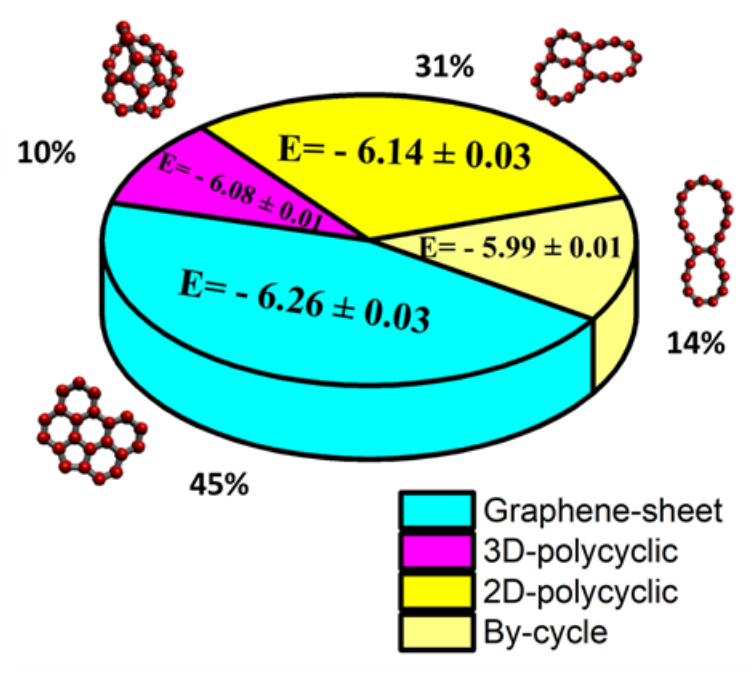

(c)

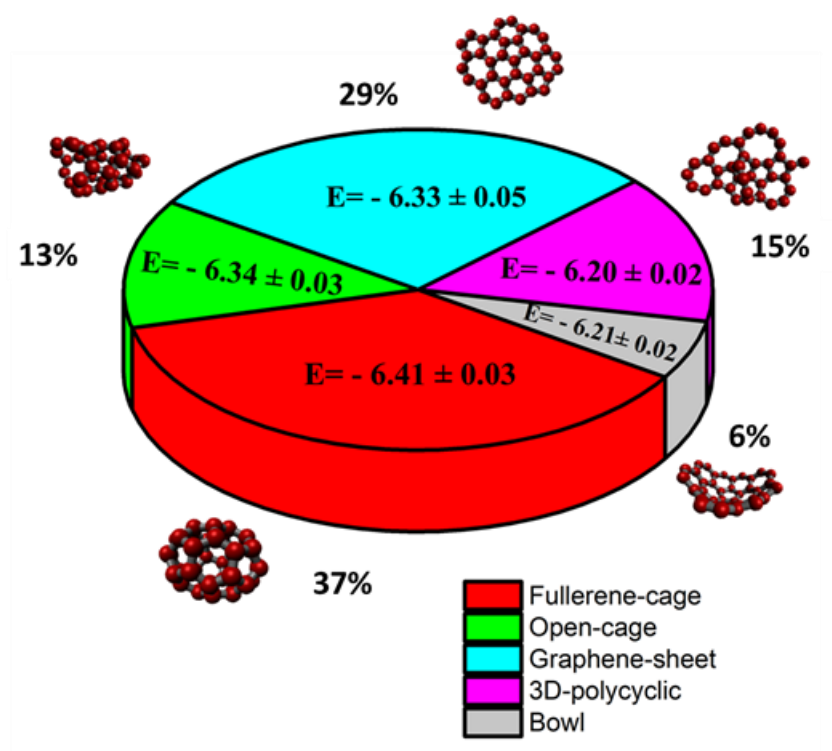

(b)

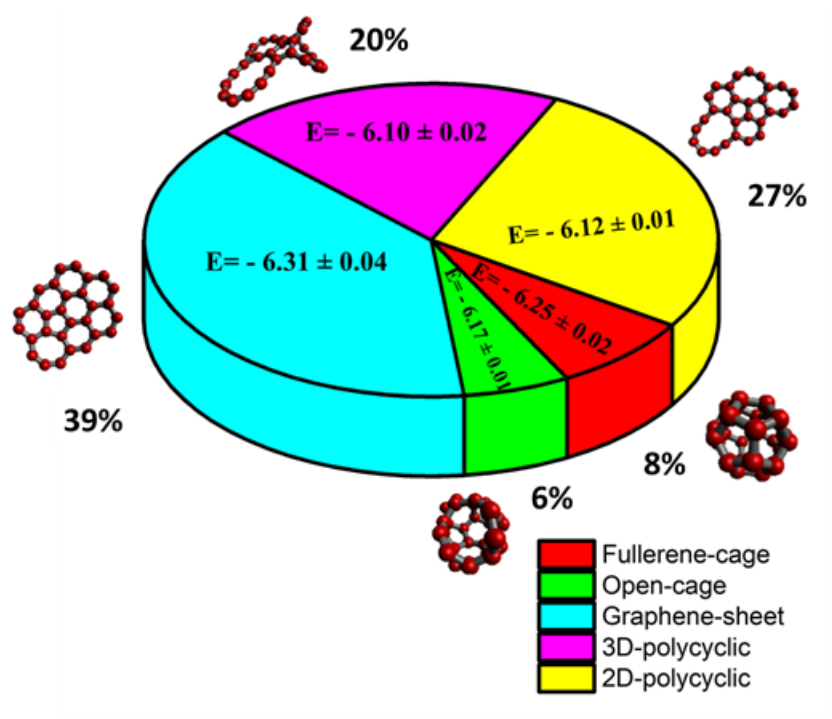

(d)

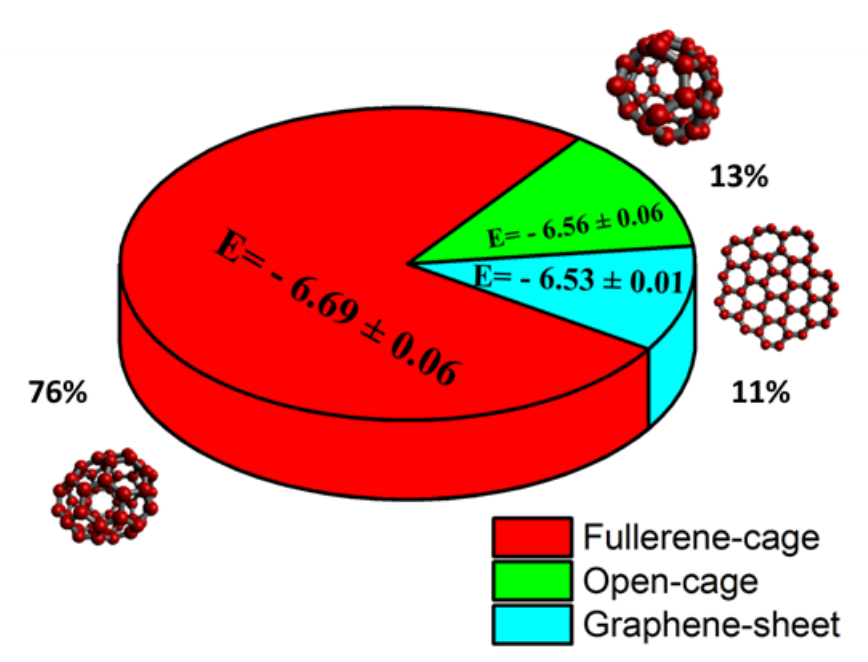

Figure 12: Statistical abundance of the six structural groups for $\mathrm{C}_{21}(\mathrm{a}), \mathrm{C}_{29}$ (b), $\mathrm{C}_{36}$ (c) and $\mathrm{C}_{50}(\mathrm{~d})$ at an annealing temperature of $3000 \mathrm{~K}$, heating and cooling times of $100 \mathrm{~ns}$ (i.e., $3 \times 10^{10} \mathrm{~K} . \mathrm{s}^{-1}$ heating and cooling rate) and a CTR phase of $100 \mathrm{~ns}$. 
We investigated the variation of the abundance of the different structural groups as function of the annealing temperature. Figure 11 shows these abundances at $2000 \mathrm{~K}, 3000 \mathrm{~K}$ and $4000 \mathrm{~K}$ for the $\mathrm{C}_{36}$ and $\mathrm{C}_{29}$ clusters.

For an annealing temperature of $2000 \mathrm{~K}, \mathrm{C}_{36}$ cluster is dominated by open cage and 2D-graphene-like structural groups, the total abundance of which is approximately $85 \%$. When the annealing temperature is increased to $3000 \mathrm{~K}$, the abundances of the different groups strongly change, and the $\mathrm{C}_{36}$ cluster becomes dominated by the closed cage structural group that represents $40 \%$ of the total population. The abundance of the open cage isomers is much smaller, $15 \%$ instead of $42 \%$ at $2000 \mathrm{~K}$, while the fraction 2D-graphene-like isomers remains significant, i.e., $28 \%$, although significantly smaller than the value obtained for $2000 \mathrm{~K}$ (40\%). Further increase of the annealing temperature to $4000 \mathrm{~K}$ leads to a structural distribution dominated by the 2D- and 3Dpolycyclic isomers that represents more than $75 \%$ of the population. Although much smaller than for lower temperature, the fraction of open-cage and 2D-graphene isomers remains significant with values of $20 \%$ and $10 \%$, respectively.

It appears therefore that the statistical abundance of the different structural form of $\mathrm{C}_{36}$ may change dramatically with the annealing temperature. Low annealing temperatures, i.e., around $2000 \mathrm{~K}$, favors graphene-like and open-cage isomers. Closed cage isomer abundance is significant at intermediate temperature; i.e., around $3000 \mathrm{~K}$. Very large annealing temperature favors the formation of polycyclic isomers. It is worthy to mention that the abundance of the graphene-like and open-cage isomers of $\mathrm{C}_{36}$ remain significant, almost $10 \%$, over the entire investigated temperature domain.

For an annealing temperature of $2000 \mathrm{~K}$, the $\mathrm{C}_{29}$ cluster is dominated by $2 \mathrm{D}$ graphene isomers, more than $60 \%$, the other structures showing abundances below $10 \%$. The increase of the temperature leads to a strong decrease of graphene-like isomers and a subsequent increase of the 3D-polycyclic and then 2D-polycyclic isomers that dominate the composition at $4000 \mathrm{~K}$. Note also, that a small fraction of closed cage structures was obtained over all the investigated range of the annealing temperature, and a small fraction, i.e., $10 \%$, of mono-ring isomers was obtained at $4000 \mathrm{~K}$. 

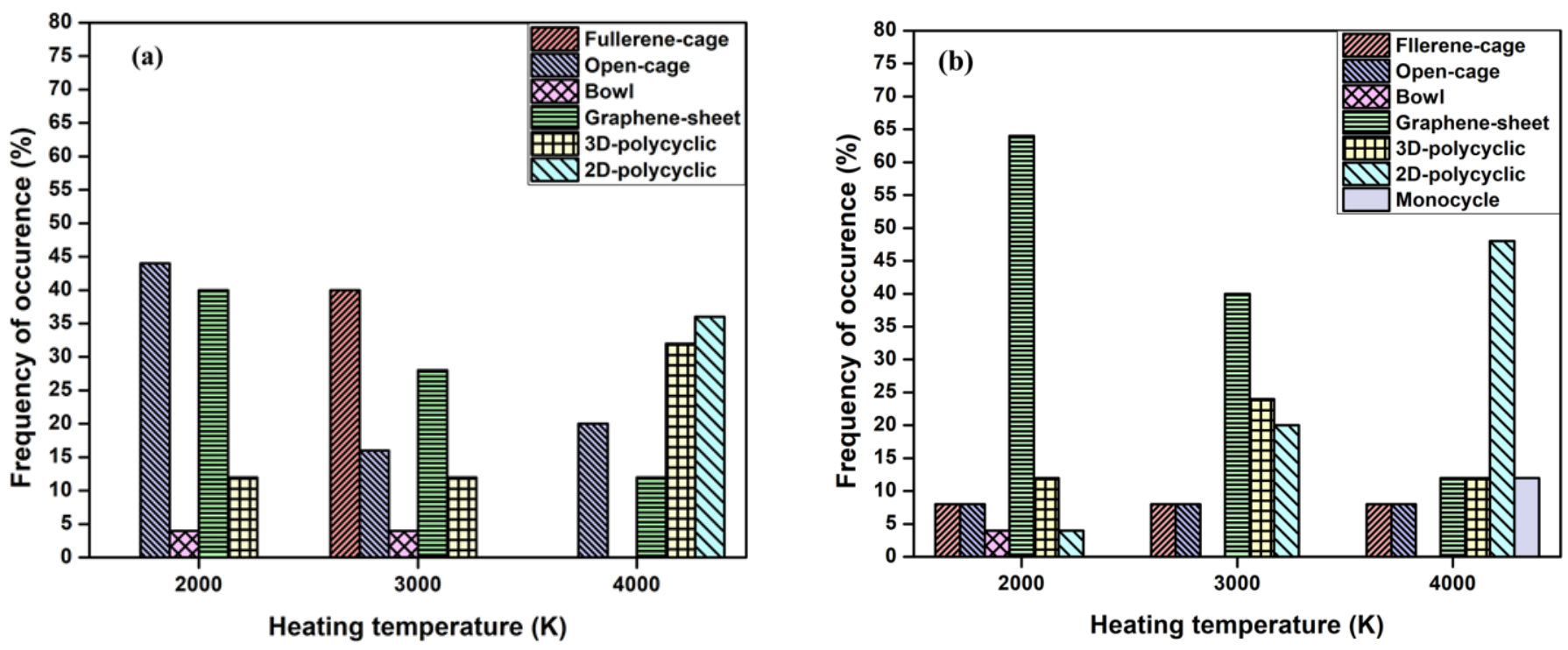

Figure 13: Evolution of the statistical abundances with the annealing temperature for $\mathrm{C}_{36}$ (a) and $\mathrm{C}_{29}$ (b) using a heating and cooling times of $100 \mathrm{~ns}$ (i.e., $3 \times 10^{10} \mathrm{~K} \cdot \mathrm{s}^{-1}$ heating and cooling rate) and a CTR phase of $100 \mathrm{~ns}$.

\section{2. c. Stable cluster isomers determined by CCA MD-simulations}

Although the CCA MD-simulations were not intended to determine the more stable structural structure of carbon clusters we find it interesting to analyze the more stable isomers determined by this CCA-MD simulation. Also interesting is to compare the more stable isomers obtained in this study and those obtained with other methods dedicated to stable structure identification. For this purpose, we conducted 25 CCA MDsimulations with optimized parameters identified in previous section (100ns/100ps/100ns and $\mathrm{T}_{\max }=3000 \mathrm{~K}$ ) for each cluster size. Typical structures obtained in these simulations are shown in Figure 12 where we also report how the cluster size is defined. The structure and energy of the most stable isomers obtained during the simulations for the different cluster sizes were recorded and are shown in Figure 15. The most stable structures obtained in our study using the SREBO potential are linear as $n$ is less than 6 . The bond lengths in $C_{n}(n=2-5)$ lie in the range of 1.310-1.331 $\AA$, which corresponds to a typical $\mathrm{C}-\mathrm{C}$ double bond. All the lowest energy linear structures calculated in our study have an outer bond length of $r_{\text {outer }}=1.310 \AA$ and an inner bond length of $r_{\text {inner }}=1.331 \AA$, which is in a good agreement with the results obtained elsewhere (Kosimov et al., 2008; Zhang et al., 2002).

When the size of carbon clusters is greater than 5 and less than $15(\mathrm{n}=6-15)$, the most stable structure obtained by CCA MD-simulation is planar mono-ring. The bond length in this structure decreases slightly from 1.364 $\AA$ to $1.341 \AA$ with increasing $n$. For $n=16$, the most stable structures are double-rings with bond lengths 
between $1.346 \AA$ and $1.439 \AA . \mathrm{C}_{17}$ and $\mathrm{C}_{18}$ have a quadruple topology with bond lengths between $1.374 \AA$ $1.454 \AA$ and $1.351 \AA-1.485 \AA$, respectively.

For $19 \leq \mathrm{n} \leq 29$, the graphene-like sheet appears to be the most stable structures. These show edge-defects consisting of pentagon or heptagon rings, except in the case of $\mathrm{C}_{27}$ and $\mathrm{C}_{28}$ which consist of only hexagon rings.

When $\mathrm{n}$ is larger than 29, the lowest energy configuration is the closed-cage structure. The most stable structures obtained using CCA MD-simulations are classical fullerenes with only hexagon and pentagon cycles for clusters with an even number of carbons, while the lowest energy structures obtained for odd size clusters are non-classical fullerenes that contain heptagon defects, as can be seen in Figure 14.

These results are consistent with those discussed in previously published works (Jones, 1999; Lai et al., 2017). The size and the width of all these clusters are defined in Figure 14 and given in Table I. The largest clusters are those with graphene-like structure and a number of carbon in the range $n=27-29$. They reach almost $1 \mathrm{~nm}$ width.

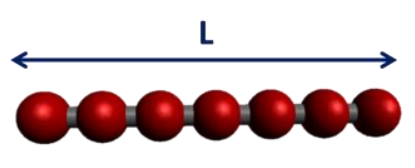

(a)

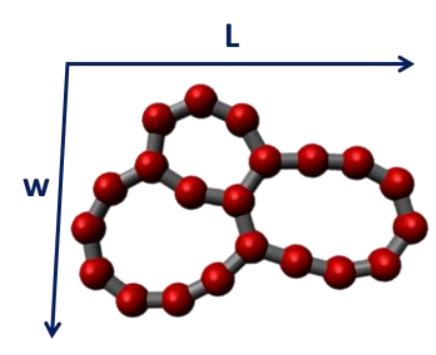

(d)

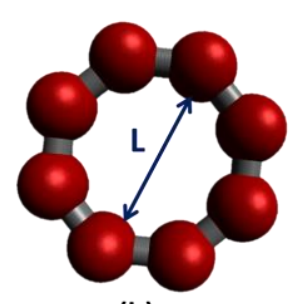

(b)

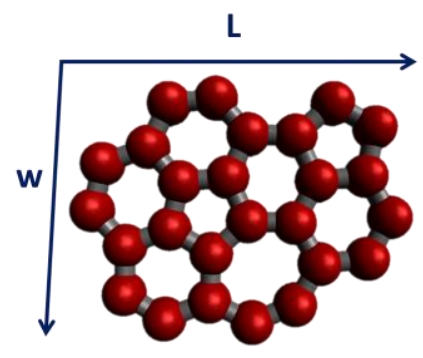

(e)

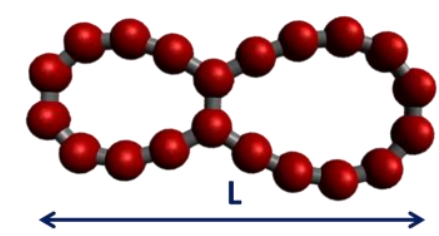

(c)

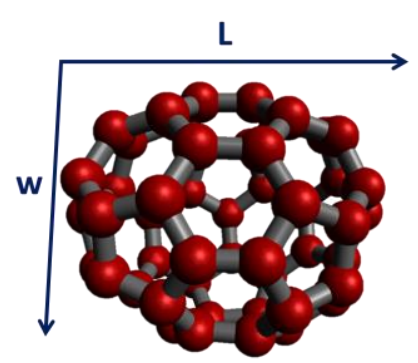

(f)

Figure 14:Typical structures obtained using CCA MD-simulations 

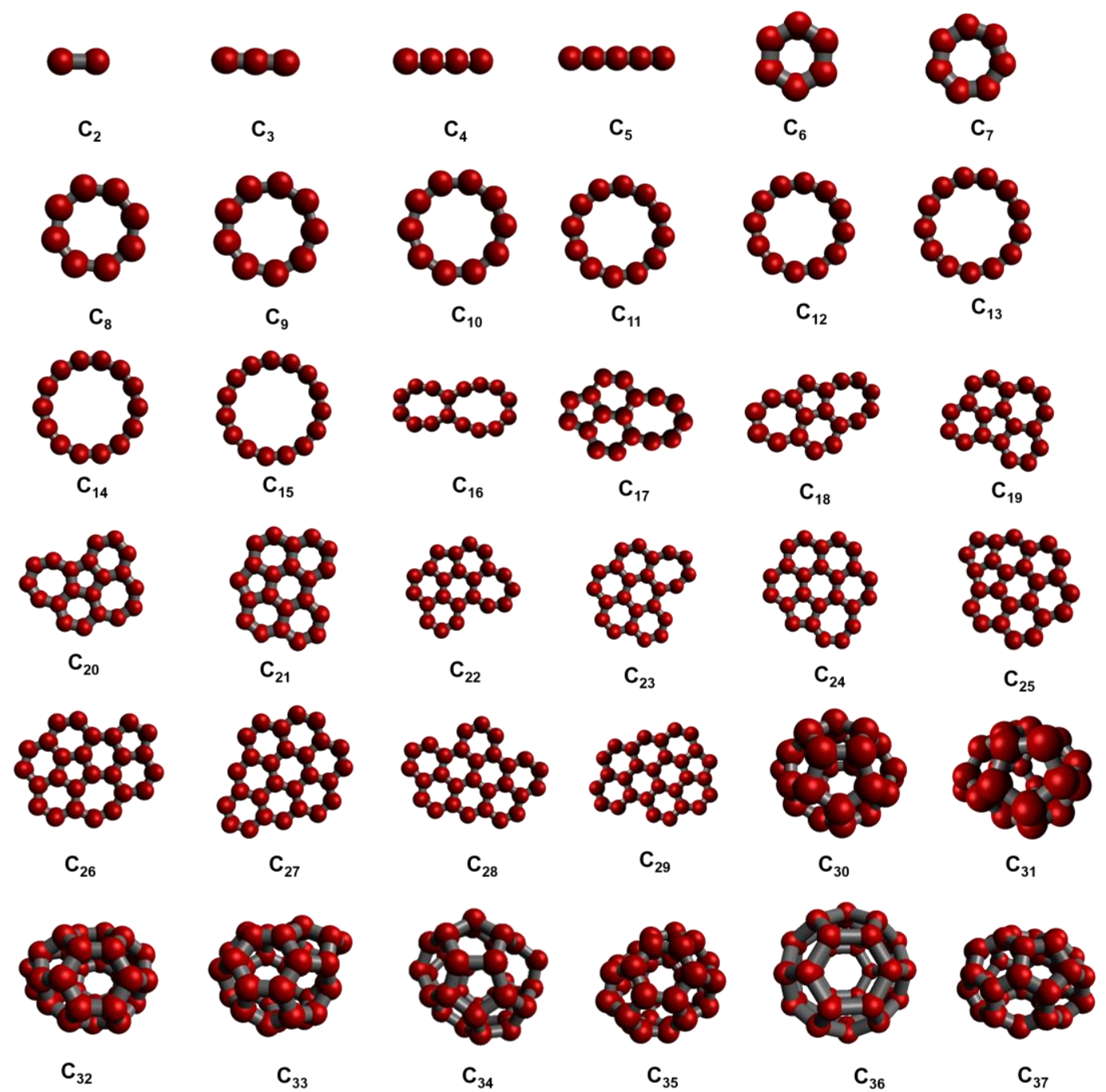

$$
\mathrm{C}_{33}
$$

$\mathrm{C}_{35}$

$\mathrm{C}_{36}$
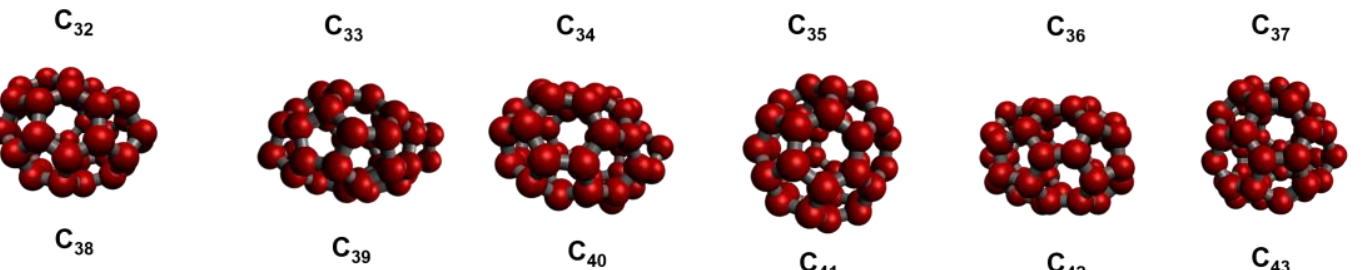

$\mathrm{C}_{42}$
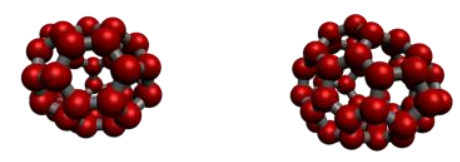

$\mathrm{C}_{45}$

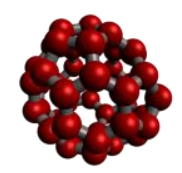

$\mathrm{C}_{46}$

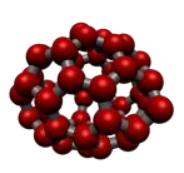

$\mathrm{C}_{47}$
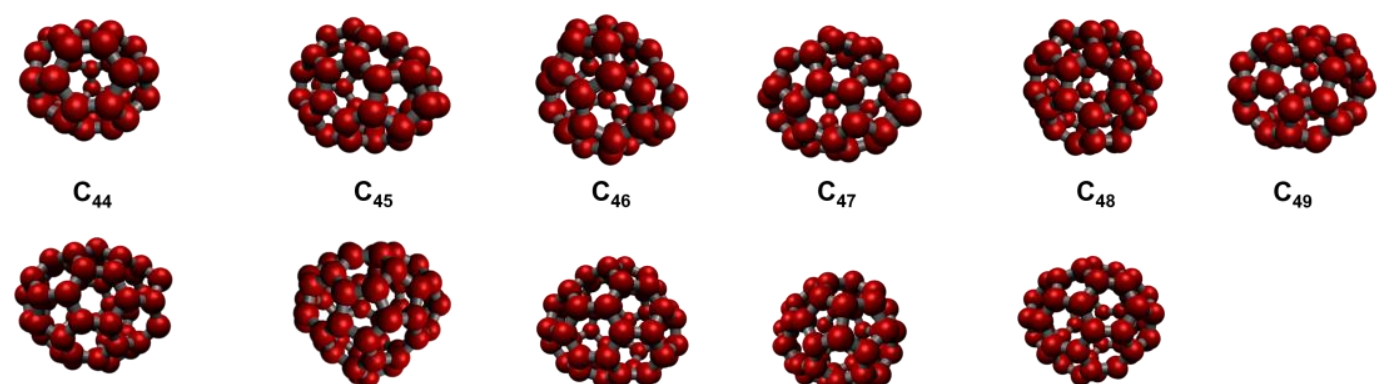

$\mathrm{C}_{50}$

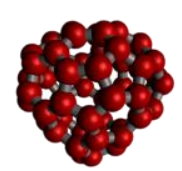

$\mathrm{C}_{51}$
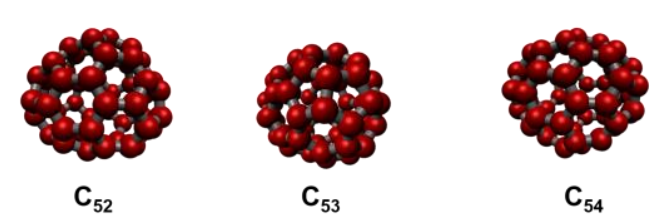

Figure 15: The most stable isomers obtained using 25 CCA simulations for cluster size between $n=2$ and $\mathrm{n}=54$. 
Table I: size bond length and bon energy of the most stable carbon clusters isomers found with CCA simulations

\begin{tabular}{|c|c|c|c|c|}
\hline $\boldsymbol{C}_{\boldsymbol{n}}$ & Bond energy & Bond length & Length $(\AA)$ & Width $(\AA)$ \\
\hline $\boldsymbol{( e V / a t o m s})$ & $(\AA)$ & & - \\
\hline $\boldsymbol{C}_{\mathbf{2}}$ & -3.10 & 1.325 & 1.325 & - \\
\hline $\boldsymbol{C}_{\mathbf{3}}$ & -4.29 & $1.310-1.331$ & 2.621 & - \\
\hline $\boldsymbol{C}_{\mathbf{4}}$ & -4.75 & $1.310-1.331$ & 3.952 & - \\
\hline $\boldsymbol{C}_{\mathbf{5}}$ & -5.03 & $1.310-1.331$ & 5.284 & - \\
\hline $\boldsymbol{C}_{\mathbf{6}}$ & -5.28 & 1.391 & 2.782 & - \\
\hline $\boldsymbol{C}_{\mathbf{7}}$ & -5.51 & 1.374 & 3.087 & - \\
\hline $\boldsymbol{C}_{\mathbf{8}}$ & -5.64 & 1.364 & 3.563 & - \\
\hline $\boldsymbol{C}_{\mathbf{9}}$ & -5.74 & 1.357 & 3.909 & - \\
\hline $\boldsymbol{C}_{\mathbf{1 0}}$ & -5.81 & 1.352 & 4.393 & - \\
\hline $\boldsymbol{C}_{\mathbf{1 1}}$ & -5.86 & 1.348 & 4.737 & - \\
\hline $\boldsymbol{C}_{\mathbf{1 2}}$ & -5.91 & 1.346 & 5.200 & - \\
\hline $\boldsymbol{C}_{\mathbf{1 3}}$ & -5.93 & 1.344 & 5.548 & - \\
\hline $\boldsymbol{C}_{\mathbf{1 4}}$ & -5.96 & 1.342 & 6.041 & - \\
\hline $\boldsymbol{C}_{\mathbf{1 5}}$ & -5.98 & 1.341 & 6.425 & - \\
\hline $\boldsymbol{C}_{\mathbf{1 6}}$ & -5.99 & $1.346-1.439$ & 8.485 & - \\
\hline $\boldsymbol{1}_{\mathbf{1 7}}$ & -5.95 & $1.374-1.454$ & 7.714 & - \\
\hline
\end{tabular}




\begin{tabular}{|c|c|c|c|c|}
\hline$C_{18}$ & -5.98 & $1.351-1.485$ & 8.561 & 4.678 \\
\hline$C_{19}$ & -6.06 & $1.361-1.483$ & 7.217 & 5.03 \\
\hline$C_{20}$ & -6.01 & $1.363-1.489$ & 8.697 & 5.039 \\
\hline$C_{21}$ & -6.27 & $1.368-1.454$ & 6.747 & 5.015 \\
\hline$C_{22}$ & -6.29 & $1.363-1.454$ & 6.651 & 4.936 \\
\hline$C_{23}$ & -6.30 & $1.354-1.457$ & 8.950 & 5.918 \\
\hline$C_{24}$ & -6.40 & $1.362-1.455$ & 8.359 & 7.312 \\
\hline$C_{25}$ & -6.39 & $1.378-1.498$ & 6.449 & 6.290 \\
\hline$C_{26}$ & -6.40 & $1.364-1.498$ & 8.836 & 6.973 \\
\hline$C_{27}$ & -6.48 & $1.367-1.431$ & 9.299 & 7.277 \\
\hline$C_{28}$ & -6.45 & $1.371-1.428$ & 9.809 & 6.956 \\
\hline$C_{29}$ & -6.31 & $1.373-1.451$ & 9.384 & 7.048 \\
\hline$C_{30}$ & -6.52 & $1.418-1.494$ & 5.859 & 4.623 \\
\hline$C_{31}$ & -6.48 & $1.417-1.492$ & 5.629 & 5.028 \\
\hline$C_{32}$ & -6.53 & $1.363-1.455$ & 5.809 & 4.612 \\
\hline$C_{33}$ & -6.56 & $1.377-1.458$ & 5.664 & 4.623 \\
\hline$C_{34}$ & -6.51 & $1.371-1.459$ & 5.926 & 4.829 \\
\hline$C_{35}$ & -6.56 & $1.417-1.498$ & 5.510 & 5.840 \\
\hline$C_{36}$ & -6.68 & $1.470-1.473$ & 5.120 & 4.570 \\
\hline$C_{37}$ & -6.62 & $1.356-1.401$ & 6.422 & 4.459 \\
\hline$C_{38}$ & -6.65 & $1.399-1.488$ & 5.539 & 5.340 \\
\hline
\end{tabular}




\begin{tabular}{|c|c|c|c|c|}
\hline$C_{39}$ & -6.53 & $1.377-1.500$ & 7.021 & 5.570 \\
\hline$C_{40}$ & -6.67 & $1.399-1.447$ & 6.823 & 5.486 \\
\hline$C_{41}$ & -6.71 & $1.382-1.483$ & 6.873 & 5.497 \\
\hline$C_{42}$ & -6.78 & $1.438-1.506$ & 6.748 & 6.459 \\
\hline$C_{43}$ & -6.76 & $1.420-1.481$ & 6.257 & 5.895 \\
\hline$C_{44}$ & -6.85 & $1.423-1.49$ & 6.861 & 6.114 \\
\hline$C_{45}$ & -6.81 & $1.412-1.503$ & 6.171 & 5.768 \\
\hline$C_{46}$ & -6.84 & $1.420-1.497$ & 6.651 & 6.531 \\
\hline$C_{47}$ & -6.74 & $1.421-1.507$ & 7.139 & 5.884 \\
\hline$C_{48}$ & -6.81 & $1.423-1.493$ & 6.809 & 6.013 \\
\hline$C_{49}$ & -6.74 & $1.431-1.489$ & 6.701 & 5.917 \\
\hline$C_{50}$ & -6.78 & $1.406-1.515$ & 6.832 & 6.438 \\
\hline$C_{51}$ & -6.74 & $1.422-1.508$ & 7.144 & 5.932 \\
\hline$C_{52}$ & -6.75 & $1.421-1.505$ & 7.448 & 6.154 \\
\hline$C_{53}$ & -6.72 & $1.421-1.498$ & 8.558 & 5.499 \\
\hline$C_{54}$ & -6.76 & $1.426-1.489$ & 6.592 & 6.461 \\
\hline$C_{55}$ & -6.74 & $1.425-1.496$ & 7.090 & 6.725 \\
\hline$C_{56}$ & -6.73 & $1.411-1.503$ & 7.318 & 6.923 \\
\hline$C_{57}$ & -6.76 & $1.414-1.498$ & 6.753 & 6.457 \\
\hline$C_{58}$ & -6.78 & $1.416-1.478$ & 8.013 & 6.864 \\
\hline$C_{59}$ & -6.79 & $1.417-1.486$ & 7.07 & 7.01 \\
\hline
\end{tabular}




\begin{tabular}{|l|l|l|l|l|}
\hline $\boldsymbol{C}_{\mathbf{6 0}}$ & -6.85 & $1.268-1.634$ & 7.778 & 6.973 \\
\hline
\end{tabular}

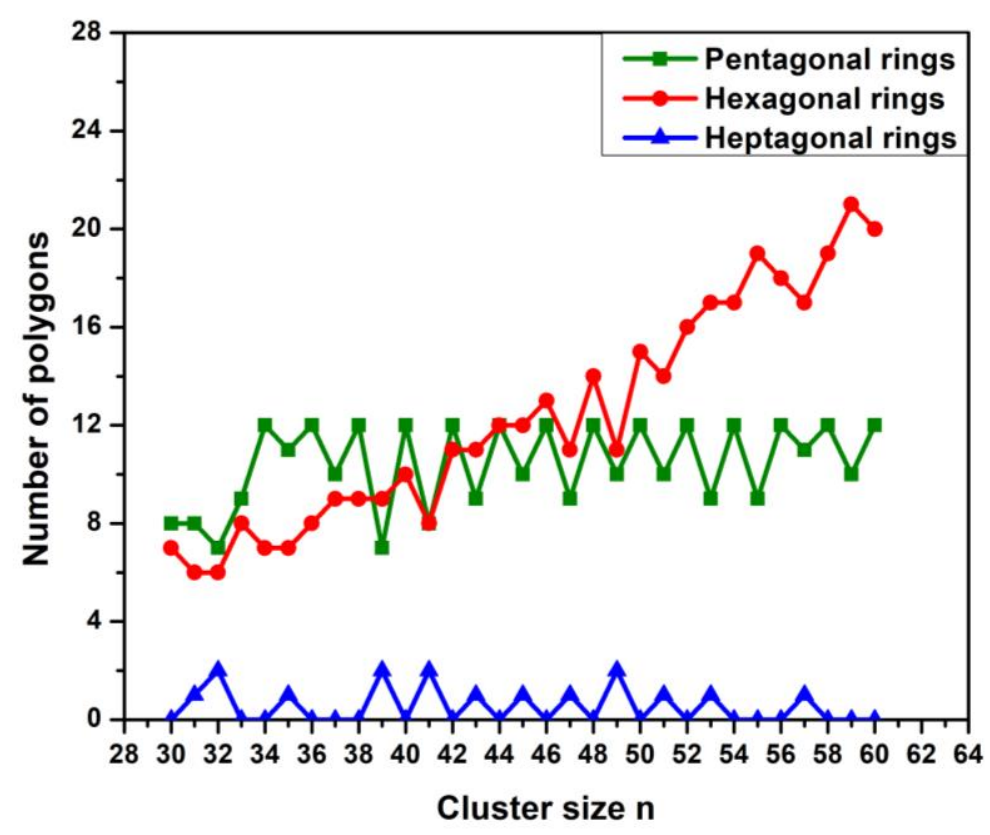

Figure 16: Number of pentagon, hexagon and heptagon in the lowest energy cage-like clusters obtained in CCA simulation. The figure shows that the lowest energy structure of even size cluster is classical fullerene clusters: 12 pentagons n/2-10 hexagons and no heptagon. The lowest energy structures of odd size clusters are non-classical fullerenes with one or two heptagons.

The energy values of the most stable structures obtained with CCA MD-simulations investigated in this work were compared to values published in the literature. Figure 17 shows the evolution of the bond energy per atom (eV/atom) determined by the CCA MD-simulations (a), MBH MC-simulations using the SREBO potential along with a modified version of the Basin-Hopping, MBH (b) (Lai et al., 2017) and DFT (LSD approximation) (c) (Jones, 1999).

All SREBO based simulations results in the same energy value for $\mathrm{C}_{2}, \mathrm{C}_{3}$ and $\mathrm{C}_{4}$ clusters. The bonding energy per atom obtained by DFT methods were shifted by a constant value so as to get the same energy value as that given by molecular dynamic simulations for $\mathrm{C}_{4}$. As a result comparison between $\mathrm{MD}$ simulations and DFT is meaningful only in terms of the relative evolutions of the bonding energy as a function of the cluster size.

CCA MD-simulations and MBH MC-simulations yield very similar energy values of the more stable structures for cluster size ranging between $n=2$ and $n=15$. DFT calculations yields similar values as MD simulations up to a cluster size of $n=8$, and slightly lower values for $n=9-15$. 
Despite the similar energy values provided by the CCA-MD and MBH-MC simulations, the structure of the most stable clusters may differ. The two methods yield linear structure for $n=3-5$ and mono-ring structure for $\mathrm{n}=6-13$. CCA-MD-simulations yield mono-ring structure for $\mathrm{C}_{14}$ and $\mathrm{C}_{15}$, while $\mathrm{MBH} \mathrm{MC}$-simulations yield a three-ring structure for $\mathrm{C}_{14}$ and a quadrupole for $\mathrm{C}_{15}$.

For a cluster size in the range $n=16-19$, the energy values of the most stable clusters obtained by CCA MD-simulations are slightly larger than the energy values obtained by MBH MC-simulations and DFT approaches. CCA MD-simulations yield double-rings structure for $\mathrm{C}_{16}$, quadrupole topology for $\mathrm{C}_{17}$ and $\mathrm{C}_{18}$, while, the MC-MBH method always yields 2D graphene-like structures in this size range. CCA MDsimulations and $\mathrm{MBH}$ MC-simulations yield 2D graphene-like structures for $\mathrm{C}_{19}$. However, while $\mathrm{MBH}-\mathrm{MC}$ yield a defect free graphene as the most stable $\mathrm{C}_{19}$ isomer, our CCA MD-simulations results in a graphene with a pentagon-heptagon pair defect, which may explain the energy difference between the most stable isomers obtained by the two methods.

For clusters with size in the range $n=20-48, C C A-M D, M B H-M C$ and DFT approaches yield very similar energy values, except for $\mathrm{C}_{29}$ and $\mathrm{C}_{43}, \mathrm{MBH}-\mathrm{MC}$ method giving slightly lower energy value for some clusters. As for the obtained structures, MBH-MC and CCA-MD methods yield closed cage structures for $\mathrm{n}=30-50$. For cluster sizes in the range 20-29, the most stable structures obtained from MBH-MC and CCAMD are very different despite their similar energy values. As a matter of fact, while CCA-MD-simulations yield graphene-like structures for $\mathrm{n}=20-29$, MBH-MC-simulations result in 2D-graphene-like structures for $\mathrm{n}=20-24$ clusters and cage like structures for $\mathrm{n}=25-29$ clusters.

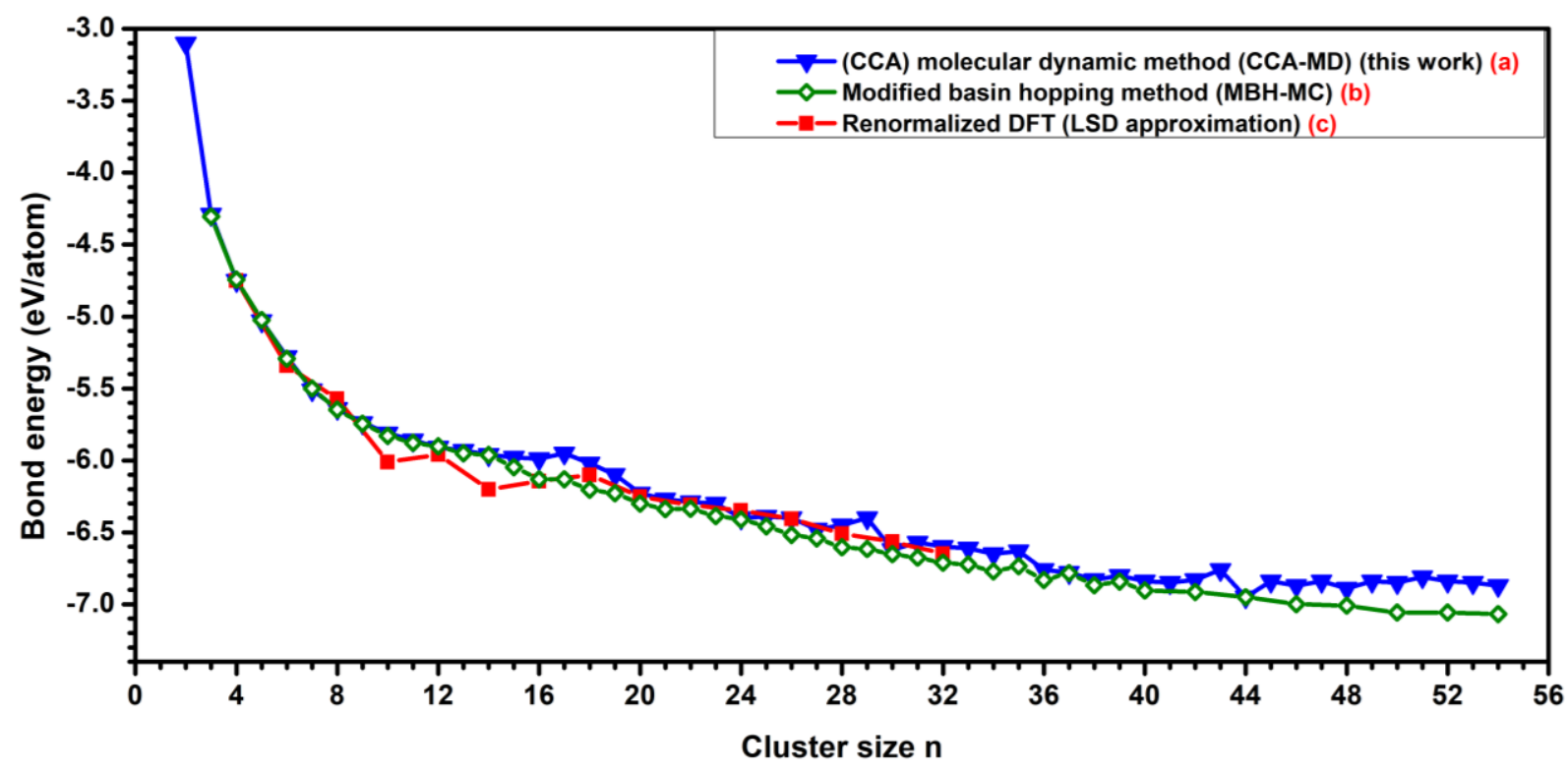

Figure 17: Variations of the bonding energy per atom, as a function of the cluster size for the most stable isomers obtained by the CCA-MD, the MBH (Lai et al., 2017) and DFT (Jones, 1999) methods. The CCA 
MD-simulations are performed using a 100ns/100ps/100ns CCA cycles at $3000 \mathrm{~K}$.

We have also compared our results with those obtained by Kosimov et al. (Kosimov et al., 2010, 2008) who used a conjugated-gradient energy minimization, CGEM, with the SREBO potential without taking into account the conjugate compensation term $\mathrm{F}_{\mathrm{ij}}\left(\mathrm{N}_{\mathrm{i}}, \mathrm{N}_{\mathrm{j}}, N_{i j}^{c o n j}\right)$ and with the objective to identify the most stable planar ring and graphene-like isomers (Kosimov et al., 2010, 2008), i.e., 3D and cage like-isomers were not considered. Figure 18-a shows, the bond energy per atom as a function of the cluster size for the most stable linear, ring or graphene structures obtained by CCA and CGEM MD-simulations, , i.e., cage like-isomers are not considered in this figure. The energy values obtained by the two methods show a very good agreement except in the size range $n=20-28$, in which the CCA MD-simulations yield slightly lower bond energy. As for the structure of the most stable clusters, CCA and CGEM-MD simulations give similar results for $\mathrm{n}=2-15$, i.e., linear structures for $n=3-5$ and mono-ring for $n=6-15$ as shown Figure 15b. The results obtained by the two approaches are however fairly different for $n=16-18$ clusters, since CGEM MD-simulations yield mono-ring structures while CCA MD-simulations give double-rings structure for $\mathrm{C}_{16}$ and quadrupole topology for $\mathrm{C}_{17}$ and $\mathrm{C}_{18}$. For $\mathrm{n} \geq 19$, the optimal planar isomers obtained by the two methods show graphene-like structures. However, the structures obtained by CGEM MD-simulations have no defects and involve only hexagons, while those obtained by CCA MD-simulations show pentagonal defects as may be seen in figure 16b. The very good agreement in the energy values obtained by CGEM and CCA MD-simulations methods for large graphene-like clusters would indicate that the approximation by Kosimov et al (Kosimov et al., 2010) in the potential expression, i.e., the omission of the conjugate compensation term, does not affect the predicted energy values for the stable graphene-like structure. This would also indicate that the significant difference in the predicted energy values for the most stable clusters by the CGEM and CCA MD simulations for $n=19-28$ is likely due to the difference in the fraction of pentagon/heptagon defects inside the graphene-like structures obtained by the two methods. As a matter of fact, the presence of even a single pentagon/heptagon edge defect in a graphene like structure at this relatively small size would have much more impact on the bond energy per atom than in the case of much larger clusters. 


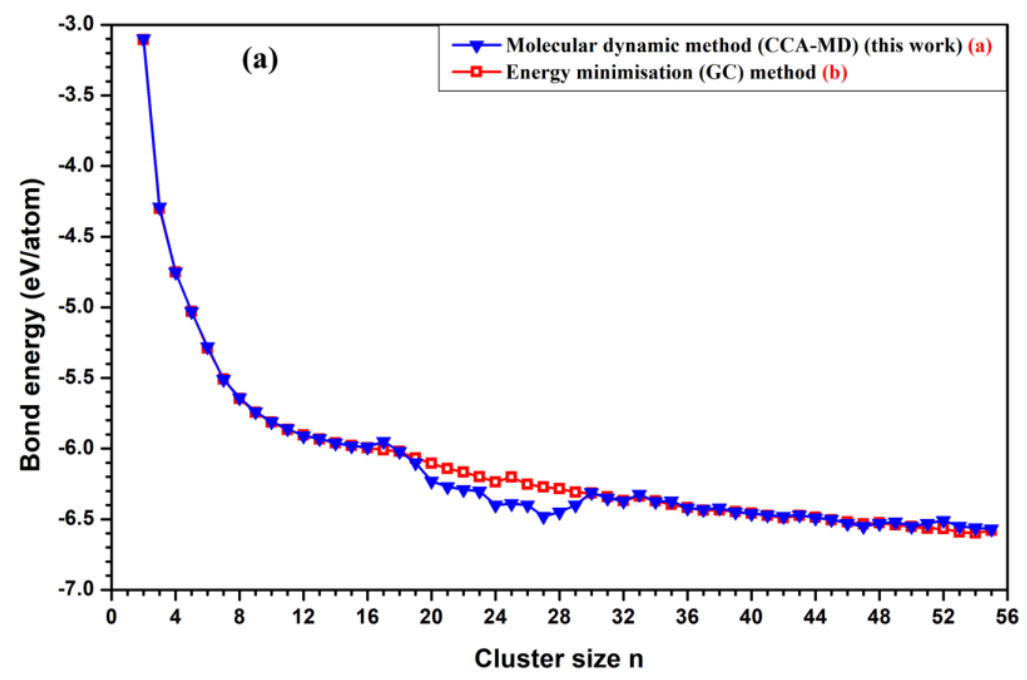

(b)

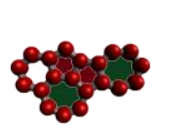

$\mathrm{C}_{20}$

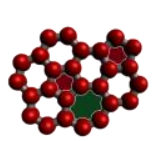

$\mathrm{C}_{26}$

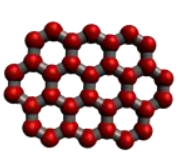

$\mathrm{C}_{32}$

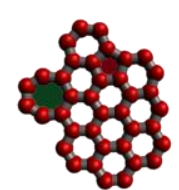

$\mathrm{C}_{38}$

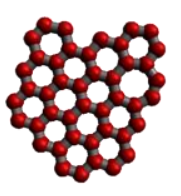

$\mathrm{C}_{44}$

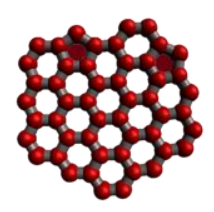

$\mathrm{C}_{50}$

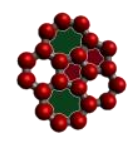

$\mathrm{C}_{21}$

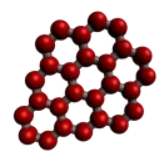

$\mathrm{C}_{27}$

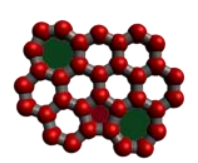

$\mathrm{C}_{33}$

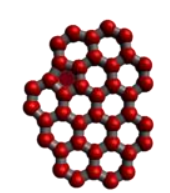

$\mathrm{C}_{39}$

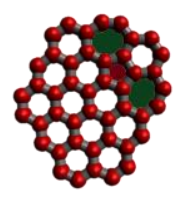

$\mathrm{C}_{45}$

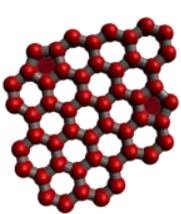

$\mathrm{C}_{51}$

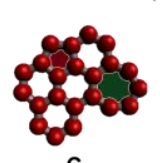

$\mathrm{C}_{22}$

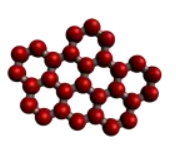

$\mathrm{C}_{28}$

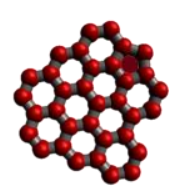

$\mathrm{C}_{34}$

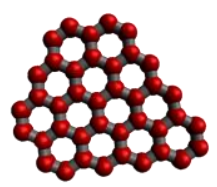

$\mathrm{C}_{40}$

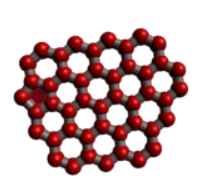

$\mathrm{C}_{46}$

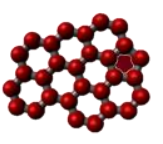

$\mathrm{C}_{29}$

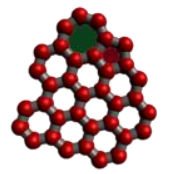

$\mathrm{C}_{35}$

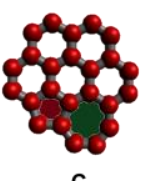

$\mathrm{C}_{24}$

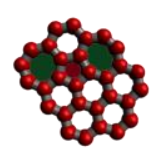

$\mathrm{C}_{30}$
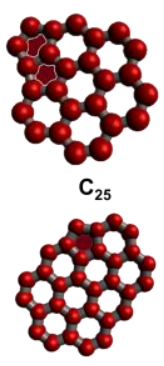

$\mathrm{C}_{31}$

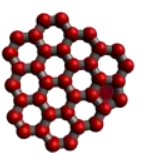

$\mathrm{C}_{36}$

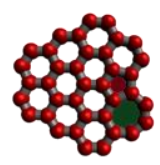

$\mathrm{C}_{37}$
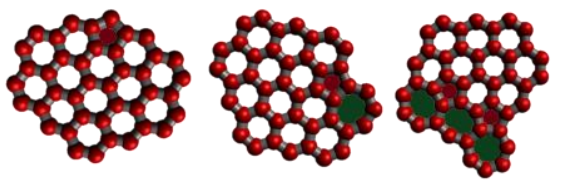

$\mathrm{C}_{41}$

$\mathrm{C}_{42}$

$\mathrm{C}_{43}$
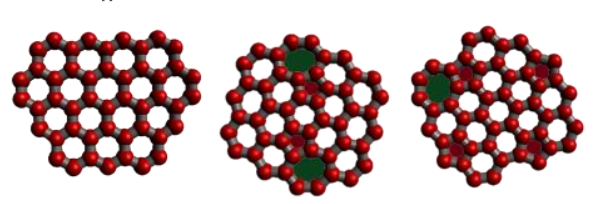

$\mathrm{C}_{47}$

$\mathrm{C}_{48}$

$\mathrm{C}_{49}$

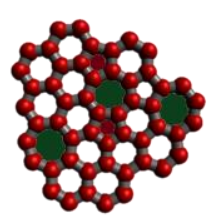

$\mathrm{C}_{52}$

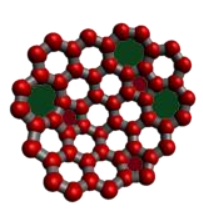

$\mathrm{C}_{53}$

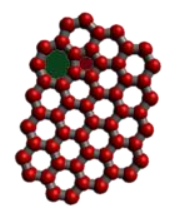

$\mathrm{C}_{54}$

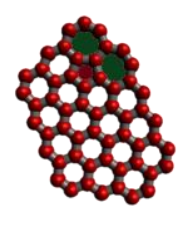

$\mathrm{C}_{55}$

Figure 18: (a) Variations of the bonding energy per atom, as a function of the cluster size for the most stable planar structures obtained by the CCA-MD and CGEM-DM (Kosimov et al., 2010, 2008) methods. (b) Graphene structures obtained by CCA MD-simulations showing pentagon (red) and heptagon (green) edgedefects. 


\section{2. d. Relative stability analysis of fullerene carbon clusters obtained by CCA MD-simulations}

Although closed cage isomers are observed for clusters as small as $\mathrm{C}_{25}$ in our CCA simulations, the closed cage isomers becomes the more stable one only when the cluster size reaches 30 . We propose therefore to examine the relative stability of the most stable fullerene isomers obtained by CCA MD-simulations as a function of the cluster size for clusters larger than $\mathrm{C}_{30}$. For this purpose, we use the second finite difference of the binding energy per atom $\Delta^{2} E(n)$ calculated as follow:

$$
\Delta^{2} E(n)=E(n-1)+E(n+1)-2 E(n)
$$

where $\mathrm{E}(\mathrm{n})$ is the energy of the most stable isomers found by our CCA MD-simulations for the cluster $\mathrm{C}_{\mathrm{n}}$. The values of $\Delta^{2} E$ as a function of $\mathrm{n}$ are shown in Figure 19 (a). The positive peaks indicate particularly stable clusters compared to their neighboring structures. The even-numbered clusters are more stable than the corresponding odd-numbered clusters. The second difference $\Delta^{2} E$ is particularly large for $\mathrm{C}_{44}$.

Figure 19 (b) shows that the occurrences of classical and non-classical fullerenes obtained by CCA MD-simulations. The classical fullerenes are obtained in the case of even-numbered clusters and appear in small frequency compared to the non-classical fullerene although they are more stable. For odd numbered clusters, only non-classical fullerene clusters are obtained.
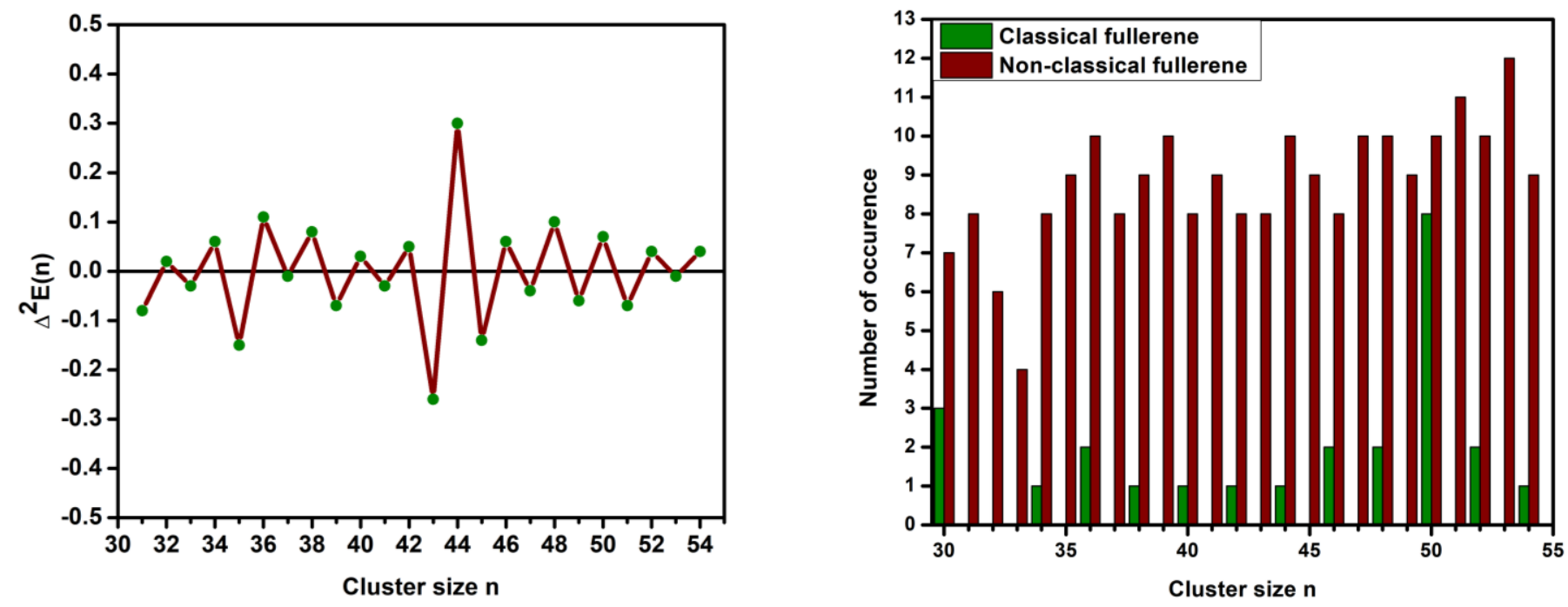

Figure 19: (a) The second finite difference of the lowest energy $\Delta^{2} \boldsymbol{E}(\boldsymbol{n})$ per atom vs cluster size n. (b) Number of classical and non-classical fullerene obtained for each cluster size $n$. 


\section{Conclusion}

Molecular Dynamics simulations of a combined condensation annealing of free carbon atoms were used in order to determine the relative abundance of different structural groups of carbon clusters as function of their size and of the annealing temperature. From the methodological point of view, we showed that the estimation of the abundances of the different structural groups requires condensation-annealing cycle with heating and CTR phase durations as long as $100 \mathrm{~ns}$ each in order to realize a full condensation of carbon atoms and relaxation of the resulting carbon clusters at the annealing temperature. On the opposite, it turns out that short CTR phase durations enable one reaching lower energy cluster structures. This is probably due to the fact that full relaxation at the annealing temperature results in the formation of the most stable cluster at this temperature. The contribution of the entropic term in the Gibbs energy that determines the stability is significant, and the most stable cluster with the lowest Gibbs energy is not necessarily the lowest energy cluster. On the opposite the entropic contribution in the Gibbs energy for cluster obtained with short CTR phase seems to be much less significant and the minimum Gibbs energy cluster would be much closer to the minimum energy cluster.

The CCA MD-simulations showed that carbon clusters may be dominated by a variety of structures depending on their size and on the considered temperature level. Further, the intermediate size clusters, i.e., $\mathrm{n}=19-36$, may show the coexistence of several, i.e., 2-3, structures at significant fractions. In any case, one may conclude that the most dominant groups for $\mathrm{n}=20-29$ clusters are $2 \mathrm{D}$-graphene-like and multi-ring structures for temperatures in the range 2000-3000 K, while multi-ring 2D and 3D structures dominate at higher temperature, i.e., $4000 \mathrm{~K}$. For clusters with $\mathrm{n} \geq 30$ open cage and 2D graphene-like structures dominate at 2000 $\mathrm{K}$, closed cage and 2D-graphene like structures at intermediate temperature, i.e., $3000 \mathrm{~K}$, and multi-ring 2D and 3D structures at higher temperature, i.e., 4000K. The fraction of closed cage clusters strongly increases with the cluster size. They represent $40 \%$ of the population for $\mathrm{C}_{36}$ and $80 \%$ of the $\mathrm{C}_{50}$ at $3000 \mathrm{~K}$. The fraction of graphene like structures remains fairly significant over all the investigated range of temperature and cluster size. For instance, $12 \%$ of the $\mathrm{C}_{50}$ clusters show Graphene-like structures at $3000 \mathrm{~K}$.

The most stable structures obtained by MD-simulations with short CTR phase CCAs were compared to the structures obtained using energy minimization methods that are much more suited to the determination of the minimum energy structures. A good agreement was obtained for the major part of the cluster in the size range 3-50. The main differences were observed in the size range $n=16-20$. The variations of the binding energy per carbon-atom of the most stable clusters obtained by CCA MD-simulations are in a good agreement with MBH MC-simulations or DFT methods. The most stable structure obtained by CCA-MD simulations are linear 
for $n=3-5$, mono-ring for $n=6-15$, multi-rings for $n=16-18$, graphene-like structure for $n=19-20$ and fullerene structure for $n \geq 30$. The majority of the most stable graphene-like structures obtained by CCA-MD simulations involve a pentagon and/or a heptagon defects. Similarly, the most stable structures found for even size number clusters are classical fullerene with only pentagon and hexagon rings, while only non-classical fullerenes, cage with one heptagon defect, were obtained for odd size number clusters.

These results have important implications as far as cluster growth kinetic through $\mathrm{C}$ and/or $\mathrm{C}_{2}$ addition under non equilibrium conditions is concerned (Armelle Michau et al., 2016; Prasanna et al., 2019). As a matter of fact, the description of this kinetic requires determining the coagulation probabilities of carbon monomer or dimer with $\mathrm{C}_{\mathrm{n}}$. For a given cluster size, n, such a probability may significantly differs depending on the considered structure, i.e., graphene like, cage-like, 3D-polycyclic, etc. The overall coagulation probability for a given cluster size may be determined from the coagulation probabilities on the different structures obtained this size and the relative predominance of these structures. The results obtained from the present work enable one setting up the methodology for investigating clustering kinetics by $\mathrm{C}$ or $\mathrm{C}_{2}$ addition. For instance, the growth of intermediate size clusters, $n=19-29$, requires considering $\mathrm{C}$ and $\mathrm{C}_{2}$ addition processes involving graphene-like, 2D-planar and 3D rings. For larger clusters, i.e., $\mathrm{n} \geq 29$, processes involving only closed cage

clusters have to be considered at temperature around $3000 \mathrm{~K}$, while processes involving graphene-like and open-cage like clusters have to be considered at lower temperature, i.e. $2000 \mathrm{~K}$.

\section{Acknowledgment}

This work was partially supported by the ANR MONA project, grant ANR-18-CE30-0016-02 of the French "Agence Nationale de la Recherche" 


\section{References}

Abell, G.C., 1985. Empirical chemical pseudopotential theory of molecular and metallic bonding. Phys. Rev. B 31, 6184-6196. https://doi.org/10.1103/PhysRevB.31.6184

AJAYAN, P., IIJIMA, S., 1992. The smallest Carbon nanotube. Nature 358, 23-23.

Alexandrov, A.L., Schweigert, V.A., n.d. A kinetic model of carbon clusters growth 4.

An, W., Gao, Y., Bulusu, S., Zeng, X.C., 2005. Ab initio calculation of bowl, cage, and ring isomers of C20 and C20-. J. Chem. Phys. 122, 204109. https://doi.org/10.1063/1.1903946

Andersen, H.C., 1980. Molecular dynamics simulations at constant pressure and/or temperature. J. Chem. Phys. 72, 2384-2393. https://doi.org/10.1063/1.439486

Arnas, C., Martin, C., Roubin, P., Pégourié, B., De Temmerman, G., Hassouni, K., Michau, A., Lombardi, G., Bonnin, X., 2010. Similarities and differences between dust produced in laboratory plasmas and in the MAST and Tore Supra tokamaks. Plasma Phys. Control. Fusion 52, 124007.

https://doi.org/10.1088/0741-3335/52/12/124007

Ballone, P., Milani, P., 1990. Simulated annealing of carbon clusters. Phys. Rev. B 42, 3201-3204. https://doi.org/10.1103/PhysRevB.42.3201

Bernholc, J., Phillips, J.C., 1986. Kinetics of aggregation of carbon clusters. Phys. Rev. B 33, 4.

Bleil, R., Tao, F.-M., Kais, S., 1994. Structure and stability of C13 carbon clusters. Chem. Phys. Lett. 229, 491494.

Brault, P., Chamorro-Coral, W., Chuon, S., Caillard, A., Bauchire, J.-M., Baranton, S., Coutanceau, C., Neyts, E., 2019. Molecular dynamics simulations of initial Pd and PdO nanocluster growth in a magnetron gas aggregation source. Front. Chem. Sci. Eng. 13, 324-329. https://doi.org/10.1007/s11705-0191792-5

Brenner, D.W., 1990. Empirical potential for hydrocarbons for use in simulating the chemical vapor deposition of diamond films. Phys. Rev. B 42, 9458.

Brenner, D.W., Shenderova, O.A., Harrison, J.A., Stuart, S.J., Ni, B., Sinnott, S.B., 2002. A second-generation reactive empirical bond order (REBO) potential energy expression for hydrocarbons. J. Phys.

Condens. Matter 14, 783-802. https://doi.org/10.1088/0953-8984/14/4/312

Cai, W., Shao, N., Shao, X., Pan, Z., 2004. Structural analysis of carbon clusters by using a global optimization algorithm with Brenner potential. J. Mol. Struct. THEOCHEM 678, 113-122.

https://doi.org/10.1016/j.theochem.2004.03.017

Dominique, C., Arnas, C., 2014. Cathode sputtering and the resulting formation of carbon nanometer-size dust. J Appl Phys 9.

Ebbesen, T.W., Tabuchi, J., Tanigaki, K., 1992. The mechanistics of fullerene formation. Chem. Phys. Lett. $191,3$.

Gan, L.-H., Liu, J., Hui, Q., Shao, S.-Q., Liu, Z.-H., 2009. General geometrical rule for stability of carbon polyhedra. Chem. Phys. Lett. 472, 224-227. https://doi.org/10.1016/j.cplett.2009.03.028

Gao, J., Lin, Z.-Z., Ning, X.-J., 2007a. Isomers of C36 and free energy criteria for cluster growth. J. Chem. Phys. 126, 174309. https://doi.org/10.1063/1.2714954

Gao, J., Lin, Z.-Z., Ning, X.-J., 2007b. Isomers of C36 and free energy criteria for cluster growth. J. Chem. Phys. 126, 174309. https://doi.org/10.1063/1.2714954

Grossman, J.C., Côté, M., Louie, S.G., Cohen, M.L., 1998. Electronic and structural properties of molecular C36. Chem. Phys. Lett. 284, 344-349. https://doi.org/10.1016/S0009-2614(97)01433-4

Harrison, B.S., Atala, A., 2007. Carbon nanotube applications for tissue engineering. Biomaterials 28, 344353. https://doi.org/10.1016/j.biomaterials.2006.07.044

Hobday, S., Smith, and, 1997. Optimisation of carbon cluster geometry using a genetic algorithm. J. Chem. Soc. Faraday Trans. 93, 3919-3926. https://doi.org/10.1039/a702961f

Jones, R.O., 1999. Density functional study of carbon clusters $C 2 n(2 \leqslant n \leqslant 16)$. I. Structure and bonding in the 
neutral clusters. J. Chem. Phys. 110, 5189-5200. https://doi.org/10.1063/1.478414

Kosimov, D.P., Dzhurakhalov, A.A., Peeters, F.M., 2010. Carbon clusters: From ring structures to nanographene. Phys. Rev. B 81, 195414. https://doi.org/10.1103/PhysRevB.81.195414

Kosimov, D.P., Dzhurakhalov, A.A., Peeters, F.M., 2008. Theoretical study of the stable states of small carbon clusters C $n(n=2-10$ ). Phys. Rev. B 78. https://doi.org/10.1103/PhysRevB.78.235433

Lai, S.K., Setiyawati, I., Yen, T.W., Tang, Y.H., 2017. Studying lowest energy structures of carbon clusters by bond-order empirical potentials. Theor. Chem. Acc. 136. https://doi.org/10.1007/s00214-016-20422

Lebedeva, I.V., Knizhnik, A.A., Bagatur'yants, A.A., Potapkin, B.V., 2008. Kinetics of 2D-3D transformations of carbon nanostructures. Phys. E Low-Dimens. Syst. Nanostructures 40, 2589-2595. https://doi.org/10.1016/j.physe.2007.09.155

Liang, C., lii, H.F.S., 1990. ELECTRONIC STRUCTUBES OF LINEAR C,, C6, Cs, AND CIO CARBON CLUSTERS AND A SYMMETRY BREAKING PHENOMENON. Chem. Phys. Lett. 169, 11.

Lichtenegger, K., von der Linden, W., 2009. Annealing Strategies in the Simulation of Fullerene Formation. ArXiv09124041 Cond-Mat.

Małolepsza, E., Witek, H.A., Irle, S., 2007. Comparison of Geometric, Electronic, and Vibrational Properties for Isomers of Small Fullerenes $\mathrm{C}_{20}-\mathrm{C}_{36}{ }^{\dagger}$. J. Phys. Chem. A 111, 6649-6657. https://doi.org/10.1021/jp068529r

Martyna, G.J., Tobias, D.J., Klein, M.L., 1994. Constant pressure molecular dynamics algorithms. J. Chem. Phys. 101, 4177-4189. https://doi.org/10.1063/1.467468

Maruyama, S., Yamaguchi, Y., 1998. A molecular dynamics demonstration of annealing to a perfect C60 structure. Chem. Phys. Lett. 286, 343-349. https://doi.org/10.1016/S0009-2614(98)00103-1

Michau, A, Arnas, C., Lombardi, G., Bonnin, X., Hassouni, K., 2016. Nanoparticle formation and dusty plasma effects in DC sputtering discharge with graphite cathode. Plasma Sources Sci. Technol. 25, 015019. https://doi.org/10.1088/0963-0252/25/1/015019

Michau, Armelle, Hassouni, K., Arnas, C., Lombardi, G., 2016. On the Competition Between the Phenomena Involved in the Aerosol Dynamics in Sputtering Nonequilibrium Plasma. IEEE Trans. Plasma Sci. 44, 528-534. https://doi.org/10.1109/TPS.2016.2519143

Ngandjong, A.C., Mezei, J.Z., Mougenot, J., Michau, A., Hassouni, K., Lombardi, G., Seydou, M., Maurel, F., 2017. Structural stability and growth mechanism of neutral and anionic small carbon clusters:

Density functional study. Comput. Theor. Chem. 1102, 105-113. https://doi.org/10.1016/j.comptc.2017.01.012

Ojwang', J.G.O., van Santen, R., Kramer, G.J., van Duin, A.C.T., Goddard, W.A., 2008. Predictions of melting, crystallization, and local atomic arrangements of aluminum clusters using a reactive force field. J. Chem. Phys. 129, 244506. https://doi.org/10.1063/1.3050278

Patra, N., Král, P., Sadeghpour, H.R., 2014. NUCLEATION AND STABILIZATION OF CARBON-RICH STRUCTURES IN INTERSTELLAR MEDIA. Astrophys. J. 785, 6. https://doi.org/10.1088/0004-637X/785/1/6

Pietrucci, F., Andreoni, W., 2014. Fate of a Graphene Flake: A New Route toward Fullerenes Disclosed with Ab Initio Simulations. J. Chem. Theory Comput. 10, 913-917. https://doi.org/10.1021/ct401103p

Plimpton, S., 1995. Fast Parallel Algorithms for Short-Range Molecular Dynamics. J. Comput. Phys. 117, 119.

Prasanna, S., Michau, A., Hassouni, K., Longo, S., 2019. Effect of charge fluctuation on nanoparticle heating in dusty plasma. Plasma Sources Sci. Technol. 28, 03LT03. https://doi.org/10.1088/13616595/ab094d

Prassides, K., Kroto, H., 1992. Fullerenes Physics. Phys. WORLD 5, 44-49.

Raghavachari, K., Binkley, J.S., 1987. Structure, stability, and fragmentation of small carbon clusters. J. Chem. Phys. 87, 2191-2197. https://doi.org/10.1063/1.453145

Samsonov, D., Goree, J., 1999. Particle growth in a sputtering discharge. J Vac Sci Technol A 17, 6. 
Schwiegert, V.A., 1995. Kinetics of carbon cluster isomerization: from tricyclic rings to fullerenes.

Sokolova, S., Lüchow, A., Anderson, J.B., 2000. Energetics of carbon clusters C20 from all-electron quantum Monte Carlo calculations. Chem. Phys. Lett. 323, 229-233.

Tang, L., Sai, L., Zhao, J., Qiu, R., 2011. Nonclassical Cn ( $n=30-40,50)$ fullerenes containing five-, six-, sevenmember rings. Comput. Theor. Chem. 969, 35-43. https://doi.org/10.1016/j.comptc.2011.05.009

Tang, Z., Wu, H., Cort, J.R., Buchko, G.W., Zhang, Y., Shao, Y., Aksay, I.A., Liu, J., Lin, Y., 2010. Constraint of DNA on Functionalized Graphene Improves its Biostability and Specificity. Small 6, 1205-1209. https://doi.org/10.1002/smll.201000024

Tersoff, J., 1989. Modeling solid-state chemistry: Interatomic potentials for multicomponent systems. Phys. Rev. B 39, 5566.

Tersoff, J., 1988. New empirical approach for the structure and energy of covalent systems. Phys. Rev. B 37, 6991.

Tersoff, J., 1986. New empirical model for the structural properties of silicon. Phys. Rev. Lett. 56, 632.

Uline, M., Corti, D., 2013. Molecular Dynamics at Constant Pressure: Allowing the System to Control Volume Fluctuations via a "Shell" Particle. Entropy 15, 3941-3969. https://doi.org/10.3390/e15093941

Wang, Y., Wei, H., Lu, Y., Wei, S., Wujcik, E., Guo, Z., 2015. Multifunctional Carbon Nanostructures for Advanced Energy Storage Applications. Nanomaterials 5, 755-777. https://doi.org/10.3390/nano5020755

Wang, Y., Zhuang, J., Ning, X.-J., 2008. Global optimization method for cluster structures. Phys. Rev. E 78, 026708. https://doi.org/10.1103/PhysRevE.78.026708

Willett, G.D., Fisher, K.J., 1992. MICHAEL A. WILSON, LOUIS S. K. PANG CSIRODivision of Coal and Energy Technology, PO Box 136, NorthRyde NSW 2 113,Australia. Carbon 30, 675-693.

Xiu-Fang, G., Yin, W., Xi-Jing, N., 2008. Growth of $C_{30}$ and $C_{31}$ Clusters: Structures, Energetics and Dynamics. Chin. Phys. Lett. 25, 468-471. https://doi.org/10.1088/0256-307X/25/2/032

Yamaguchi, Y., Maruyama, S., 1998. A molecular dynamics simulation of the fullerene formation process. Chem. Phys. Lett. 286, 336-342. https://doi.org/10.1016/S0009-2614(98)00102-X

Zhang, C., Xu, X., Wu, H., Zhang, Q., 2002. Geometry optimization of $\mathrm{Cn}(\mathrm{n}=2-30)$ with genetic algorithm. Chem. Phys. Lett. 364, 213-219. https://doi.org/10.1016/S0009-2614(02)01338-6 
